\title{
The XMM-LSS survey: optical assessment and properties of different X-ray selected cluster classes ${ }^{\star}$
}

C. Adami ${ }^{1}$, A. Mazure ${ }^{1}$, M. Pierre ${ }^{2}$, P. G. Sprimont ${ }^{3}$, C. Libbrecht ${ }^{2}$, F. Pacaud ${ }^{2,8}$, N. Clerc ${ }^{2}$, T. Sadibekova ${ }^{3}$, J. Surdej ${ }^{3}$, B. Altieri ${ }^{4}$, P. A. Duc ${ }^{2}$, G. Galaz ${ }^{7}$, A. Gueguen ${ }^{2}$, L. Guennou ${ }^{1}$, G. Hertling ${ }^{7}$, O. Ilbert ${ }^{1}$, J. P. Le Fèvre ${ }^{14}$, H. Quintana ${ }^{7}$, I. Valtchanov ${ }^{4}$, J. P. Willis ${ }^{9}$, M. Akiyama ${ }^{12}, \mathrm{H}_{\text {. Aussel }}^{2}$, L. Chiappetti $^{10}$, A. Detal $^{3}$, B. Garilli ${ }^{10}$, V. LeBrun ${ }^{1}$, O. LeFèvre ${ }^{1}$, D. Maccagni ${ }^{10}$, J. B. Melin ${ }^{13}$, T. J. Ponman ${ }^{11}$, D. Ricci ${ }^{3}$, and L. Tresse ${ }^{1}$

${ }^{1}$ LAM, OAMP, Pôle de l'Etoile Site Château-Gombert 38, Rue Frédréric Juliot-Curie, 13388 Marseille Cedex 13, France e-mail: christophe.adami@oamp.fr

2 Laboratoire AIM, CEA/DSM/IRFU/Sap, CEA-Saclay, 91191 Gif-sur-Yvette Cedex, France

3 Institut d'Astrophysique et de Géophysique, Université de Liège, Allée du 6 Août 17, B5C, 4000 Sart Tilman, Belgium

4 ESA, Villafranca del Castillo, Spain

5 UPMC Université Paris 06, UMR 7095, Institut d'Astrophysique de Paris, 75014 Paris, France

6 CNRS, UMR 7095, Institut d'Astrophysique de Paris, 75014 Paris, France

7 Departamento de Astronomía y Astrofísica, Pontificia Universidad Católica de Chile, Casilla 306, Santiago 22, Chile

8 Argelander-Institut für Astronomie, University of Bonn, Auf dem Hügel 71, 53121 Bonn, Germany

9 Department of Physics and Astronomy, University of Victoria, Elliot Building, 3800 Finnerty Road, Victoria, V8V 1A1, BC, Canada

10 INAF-IASF Milano, via Bassini 15, 20133 Milano, Italy

11 School of Physics and Astronomy, University of Birmingham, Edgbaston, Birmingham, B15 2TT, UK

12 Astronomical Institute, Tohoku University 6-3 Aramaki, Aoba-ku, Sendai 980-8578, Japan

${ }^{13}$ CEA/DSM/IRFU/SPP, CEA Saclay, 91191 Gif-sur-Yvette, France

14 CEA/DSM/IRFU/SEDI, CEA Saclay, 91191 Gif-sur-Yvette, France

Received 9 June 2010 / Accepted 28 October 2010

\begin{abstract}
Context. XMM and Chandra opened a new area for the study of clusters of galaxies not only for cluster physics, but also for the detection of faint and distant clusters that were inaccessible with previous missions.

Aims. This article presents 66 spectroscopically confirmed clusters $(0.05 \leq z \leq 1.5)$ within an area of 6 deg $^{2}$ enclosed in the XMM-LSS survey. Almost two thirds have been confirmed with dedicated spectroscopy only and $10 \%$ have been confirmed with dedicated spectroscopy supplemented by literature redshifts.

Methods. Sub-samples, or classes, of extended-sources are defined in a two-dimensional X-ray parameter space allowing for various degrees of completeness and contamination. We describe the procedure developed to assess the reality of these cluster candidates using the CFHTLS photometric data and spectroscopic information from our own follow-up campaigns.

Results. Most of these objects are low-mass clusters, hence constituting a still poorly studied population. In a second step, we quantify the correlations between the optical properties such as richness or velocity dispersion and the cluster X-ray luminosities. We examine the relation of the clusters to the cosmic web. Finally, we review peculiar compact structures in the surveyed area such as very distant clusters and fossil groups.
\end{abstract}

Key words. surveys - galaxies: clusters: general - large-scale structure of Universe - X-rays: galaxies: clusters

\footnotetext{
* Based on observations obtained with MegaPrime/MegaCam, a joint project of CFHT and CEA/DAPNIA, at the Canada-France-Hawaii Telescope (CFHT), which is operated by the National Research Council (NRC) of Canada, the Institut National des Science de l'Univers of the Centre National de la Recherche Scientifique (CNRS) of France, and the University of Hawaii. This work is based in part on data products produced at TERAPIX and the Canadian Astronomy Data Centre as part of the Canada-France-Hawaii Telescope Legacy Survey, a collaborative project of NRC and CNRS. This work is also based on observations collected at TNG (La Palma, Spain), Magellan (Chile), and at ESO Telescopes at the La Silla and Paranal Observatories under programmes ID 072.A-0312, 074.A-0476, 076.A-0509, 070.A-0283, 072.A-0104, and 074.A-0360.
}

\section{Introduction}

With the quest for the characterization of the Dark Energy properties and the upcoming increasingly large instruments (JWST, ALMA, LSST, EUCLID, etc.) the beginning of the 21st century will be an exciting time for cosmology. In this respect, a new era was already opened for X-ray astronomy by the XMM-Newton and Chandra observatories in 1999. The increasing amount of high-quality multi-wavelength observations and the concept of a "multi-probe" approach is expected to provide strong constraints on the cosmological models. In this context, X-ray surveys have an important role to play, as it was already the case in the $1980 \mathrm{~s}$ and $1990 \mathrm{~s}$ (e.g. Romer et al. 1994; Castander et al. 1995; Collins et al. 1997; Henry et al. 1997; Bohringer et al. 1998; Ebeling et al. 1998; Jones et al. 1998; Rosati et al. 1998; 
Vikhlinin et al. 1998; De Grandi et al. 1999; Romer et al. 2000, and Ref. therein). New cluster surveys are constantly set in motion (e.g. Romer et al. 2001; Pierre et al. 2004; Finoguenov et al. 2007).

One of them, the XMM-LSS survey, covers $11 \mathrm{deg}^{2}$ at a sensitivity of $\sim 10^{-14} \mathrm{erg} / \mathrm{s} / \mathrm{cm}^{2}$ at $0.5-2 \mathrm{keV}$ for spatially-extended $\mathrm{X}$-ray sources and is currently the largest contiguous deep XMM cluster survey. This sky region is covered by parallel surveys in multiple complementary wavebands ranging from radio to the $\gamma$-ray wavelengths (Pierre et al. 2004) and therefore constitutes a unique area for pioneering studies. It can for instance detect a Coma-like cluster at $z \sim 2$. A number of articles describing the properties of the XMM-LSS source population have been published by e.g. Pierre et al. (2006) and Pacaud et al. (2007) for clusters of galaxies and Gandhi et al. (2006) for AGNs; the complete X-ray source catalog along with optical identifications for the first $5 \mathrm{deg}^{2}$ of the survey was published by Pierre et al. (2007).

One of the major goals of the XMM-LSS survey is to provide samples of galaxy clusters with well defined selection criteria to enable cosmological studies out to redshift $z \sim 1.5$. Indeed, monitoring selection effects is mandatory not only to study the evolution of the cluster X-ray luminosity (i.e. mass) function or of the 3-D cluster distribution but also, as shown by Pacaud et al. (2007), to characterize the evolution of the cluster scaling laws such as the luminosity-temperature relation. We have put special emphasis on the X-ray selection criteria in the XMM-LSS survey. The procedure enables the construction of samples with various degrees of completeness and allows for given rates of contamination by non-cluster sources. The subsequent optical spectroscopic observations constitute the ultimate assessment of the clusters.

In a first paper, Pacaud et al. (2007) presented the Class One (C1) clusters pertaining to the first $5 \mathrm{deg}^{2}$ of the survey (the ones with the highest a priori probability to be real clusters). The C1 selection yields a purely X-ray selected cluster sample with an extremely low contamination level and corresponds to fairly high surface brightness objects. The present article summarizes these former findings including now the clusters selected from less stringent $\mathrm{X}$-ray criteria (C2 and C3) and including the contiguous Subaru Deep Survey (SXDS, e.g. Ueda et al. 2008). The $\mathrm{C} 2$ and $\mathrm{C} 3$ objects presented here come from an initial sample with a higher degree of contamination, but have all passed the final spectroscopic tests. Compared to the $\mathrm{C} 1$ clusters, they are fainter and correspond a priori to less massive clusters or to groups at a redshift of $\sim 0.5$ : this is a population that is for the first time systematically unveiled by the XMM-LSS survey. A few massive very distant clusters are falling into this category too.

The present study is the first attempt to give a comprehensive census (X-ray and optical properties) of the low-mass cluster population within the $0<z<1$ range. The search for correlations between optical and X-ray properties has already been going on a long time, since e.g. Smith et al. (1979) or Quintana \& Melnick (1982). However, with more than 60 spectroscopically confirmed clusters, the current sample constitutes by far the spectroscopically confirmed cluster sample with the highest surface density ever published. The article is organized as follows. In the next section we describe the X-ray cluster selection. Section 3 presents the available optical photometric and spectroscopic data. Section 4 explains the adopted cluster validation procedure, the new X-ray luminosity computations, and presents the resulting catalog. Then, the global properties of each cluster class and category are examined in Sect. 5 and, subsequently, the properties of the cluster galaxy population in Sect. 6. Section 7 details the $z=1.53$ candidate cluster and investigates possible peculiar compact structures in the survey. Finally Sect. 8 presents the conclusions. The two appendices discuss the accuracy of photometric redshifts in the context of dense environments and lists additional redshift structures found in the course of the study.

Throughout the paper we assume $H_{0}=71 \mathrm{~km} \mathrm{~s}^{-1} \mathrm{Mpc}^{-1}$, $\Omega_{\mathrm{m}}=0.27$, and $\Omega_{\Lambda}=0.73$ (Dunkley et al. 2009). All magnitudes are in the $A B$ system.

\section{The initial cluster candidate selection}

The clusters presented in this paper are for the great majority X-ray-selected. The XMM-LSS pipeline (Pacaud et al. 2006) provides some 20 parameters for each detected source (coordinates, count rate, etc.). Out of these, two are especially relevant for the characterization of extended sources: the extent measurement (EXT) and the likelihood of extent (EXT_LH). We recall (as defined by Pacaud et al. 2006) that the "extent" parameter is the core radius of the beta-profile fit by the survey pipeline to each source, assuming a fixed beta of $2 / 3$. The cluster selection basically operates in this two-dimensional space and has been extensively adjusted and tested using simulations of hundreds of XMM images. This allows the definition of three cluster samples.

- the $\mathrm{C} 1$ class is defined such that almost no point sources are misclassified as extended (i.e. less than $1 \%$ of the cluster candidates are point sources) and is described by EXT $>5^{\prime \prime}$, EXT_LH $>33$ plus an additional boundary on the detection likelihood, set to be greater than 32 ;

- the C2 class is limited by EXT $>5^{\prime \prime}$, EXT_LH $>15$ and displays an a priori contamination rate of about $50 \%$;

- the C3 clusters are faint objects and therefore have less-well characterized X-ray properties. They may be located at the very edge of the XMM field of view or suffer contamination by point sources. They therefore result from a subjective selection mostly based on a visual inspection of the X-ray and optical data; their selection function is up to now undefined.

More details about the classification can be found in Pacaud et al. (2006) and Pierre et al. (2006).

We present a large sample of X-ray clusters, including the 29 C1 confirmed clusters published by Pacaud et al. (2007). These $\mathrm{C} 1$ clusters were already unambiguously confirmed, but we take the occasion of this publication to reprocess the associated optical spectroscopic data following the standard method developed in the present paper. This will provide a unique homogeneous cluster sample. The clusters pertaining to this paper are mostly located in the first $5 \mathrm{deg}^{2}$ of the XMM-LSS region, supplemented by the Subaru Deep Survey. The validated C1, C2, and C3 samples are presented in Tables 2-4. In these tables, XLSS catalog names refer to sources published in Pierre et al. (2007). XLSSU catalog names refer to sources whose fields were not yet considered in XLSS (for example flagged bad or in SXDS fields) and reobserved (or reprocessed later), or which were below the detection likelihood threshold in the input data set used as source for XLSS.

In the course of the data inspection, we also identified a few clusters using optically based criteria such as the red sequence or the gapper method. Our spectroscopic data set allowed us to confirm them as bona fide clusters, although these objects are not detected in the X-rays by the current version of the XMM-LSS 
Table 1. PI spectroscopic runs involved in the present paper.

\begin{tabular}{ccccl}
\hline \hline Telescope & Instrument & Year & Nights & Run ID \\
\hline Magellan & LDSS2 & 2002 & 2 & - \\
Magellan & LDSS2 & 2003 & 4 & - \\
NTT & EMMI & 2003 & 3 & $72 . \mathrm{A}-0312$ \\
NTT & EMMI & 2004 & 4 & 74.A-0476 \\
NTT & EMMI & 2005 & 3 & 76.A-0509 \\
TNG & DOLORES & 2007 & 4 & AOT16/CAT_75 \\
VLT & FORS2 & 2002 & 3 & 70.A-0283 \\
VLT & FORS2 & 2003 & 4 & 72.A-0104 \\
VLT & FORS2 & 2004 & 4.5 & 74.A-0360 \\
\hline
\end{tabular}

pipeline or the association between X-ray detected sources and optical clusters is not straightforward. We list these objects in Tables 5, and B.1.

We now describe the involved optical data and the general identification processes.

\section{The optical data}

\subsection{The optical spectroscopic data}

We have been performing a dedicated spectroscopic follow-up of all $\mathrm{C} 1$ clusters and of a number of $\mathrm{C} 2$ and $\mathrm{C} 3$ clusters. These PI observations are listed in Table 1 and provide about 2000 redshifts to date. We supplemented this data set with the VVDS deep (e.g. Le Fèvre et al. 2005: 11000 redshifts in $0.49 \mathrm{deg}^{2}$ ) and ultradeep (LeFèvre et al., in prep.) data, and with a redshift compilation pertaining to the Subaru Deep Survey (Ueda et al. 2008) included in the XMM-LSS area. Some 200 other redshifts were also available from the NASA/IPAC Extragalactic Database (NED hereafter) for part of the area. We show in Fig. 1 the location of these different surveys, and the exposure time of the different XMM fields.

Individual redshift measurements of spectra resulting from the PI data were made following a procedure similar to that adopted by the VVDS survey. Each spectrum was independently measured by several people, and the redshift was subsequently validated by a moderator. Quality flags were assigned to each measurement following the VVDS rules: flag 0 indicates an inconclusive result, flag 1 means a probability of $50 \%$ that the assigned redshift is wrong, flag 2 means a probability of $25 \%$, flag 3 means a probability of $5 \%$, flag 4 means a probability of $1 \%$, and flag 9 means we have assigned a redshift with a single line using absent lines in order to limit the possibilities. These percentage levels proved to be reliable in the VVDS survey (Le Fèvre et al. 2005).

Because our spectroscopic redshifts have quite heterogeneous origins (different telescopes, instrumentations, and resolutions), it is useful to compute the ability to measure a redshift and the achieved velocity resolution. In order to achieve such a goal, we chose to compare the PI data to the VVDS survey, which provides a well qualified set of data. Only 26 galaxies measured by both the VVDS and our dedicated follow-up have a quality flag greater than or equal to 2 . For these objects, given the VVDS quality flags (6 flags 2, 6 flags 3, and 14 flags 4), we expect to have 3.2 wrong redshifts. We indeed find 3 redshifts differing by more than 0.05 between the PI and VVDS data. VVDS spectroscopic redshifts are expected to have a typical uncertainty of $280 \mathrm{~km} \mathrm{~s}^{-1}$ (from repeated VVDS redshift measurements, Le Fèvre et al. 2005). Excluding all redshifts with differences greater than 0.02 , we find a typical uncertainty between PI and VVDS redshifts of $340 \mathrm{~km} \mathrm{~s}^{-1}$. Even with a comparison done

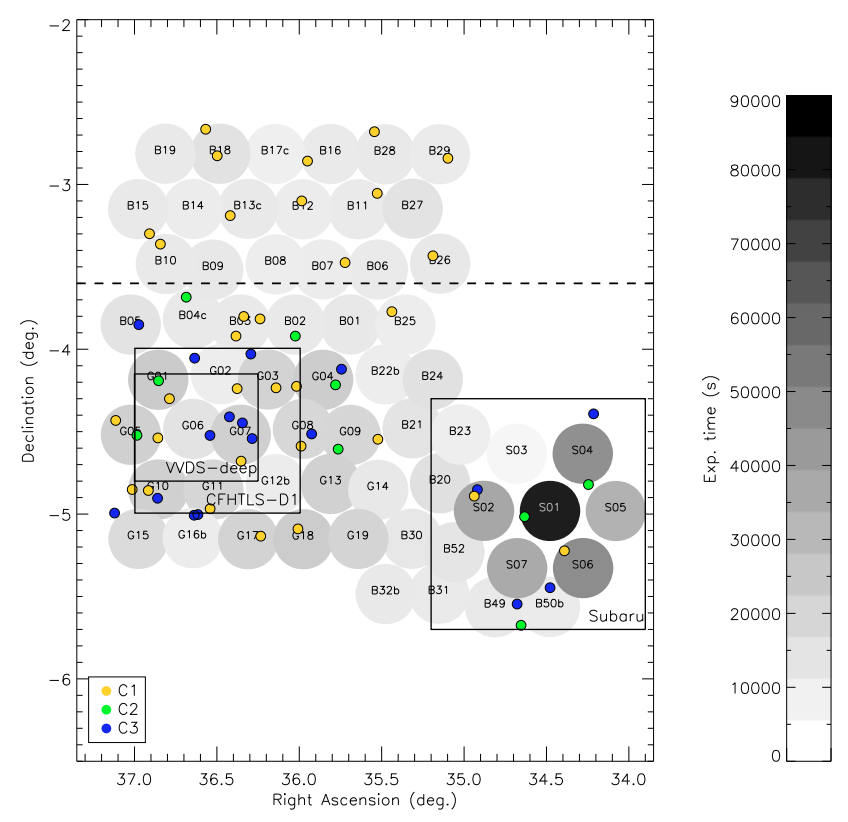

Fig. 1. Map showing the different involved surveys. The gray level disks are the $11^{\prime}$ central areas of the XMM pointings (exposure time depends on the greyness). Large squares show the spectroscopic VVDS-deep and Subaru Deep surveys, and the CFHTLS D1 field. C1, C2, and C3 clusters are also shown. Above a declination of $-3.6 \mathrm{deg}$, only $g^{\prime}, r^{\prime}, z^{\prime}$ coverage is available, hence no photometric redshifts are derived for this zone.

on a somewhat limited size sample, the PI redshifts appear thus reliable in the [0.,1.] redshift range and in the [18,23] I VVDS magnitude range.

Finally, it has to be mentioned that for the spectroscopic sample no completeness, either spatial or in luminosity, can be generally defined because of the various data origins.

\subsection{The optical photometric data}

Most of the XMM-LSS area is covered by the Canada-FranceHawaii Telescope Legacy Wide Survey (CFHTLS-Wide ${ }^{1}$ ). This survey, performed by means of the MegaCam camera, covers some $171 \mathrm{deg}^{2}$ in four independent patches with five filters $\left(u^{*}, g^{\prime}, r^{\prime}, i^{\prime}\right.$ and $\left.z^{\prime}\right)$. Resulting catalogs are $80 \%$ complete down to $i_{\mathrm{AB}}^{\prime}=24$. The Wide survey encloses a sample of about $20 \times 10^{6}$ galaxies inside a volume size of $\sim 1 \mathrm{Gpc}^{3}$, with a median redshift of $z \sim 0.92$ (Coupon et al. 2009). Northern of Dec $=-3.6 \mathrm{deg}$, the CFHTLS data were complemented by PI MegaCam observations $\left(3 \mathrm{deg}^{2}\right)$ performed in $g^{\prime}, r^{\prime}, z^{\prime}$ at the same depth as the CFHTLS; they were reduced following the same procedure.

The optical images and catalogs were primarily used to check for galaxy concentrations coinciding with the extended X-ray emission. The CFHTLS data (only the T0004 release was available at the beginning of the present study) enabled the determination of photometric redshifts in the best-fit template (Coupon et al. 2009). These photometric redshifts cover $35 \mathrm{deg}^{2}$ in the T0004 partially overlapping with the XMM-LSS area. They were computed using a template-fitting method, calibrated with public spectroscopic catalogs. The method includes

${ }^{1}$ http://terapix.iap.fr/cplt/T0006/T0006-doc.pdf 
correction of magnitude systematic offsets. The achieved photometric redshift precision $\sigma_{z} /(1+z)$ is on the order of 0.04 with a catastrophic error percentage of less than $5 \%$ at $i^{\prime} \leq 23$ (the magnitude limit we adopted for the photometric redshifts).

\section{4. "Cluster candidate" validation process}

\subsection{General method}

Extragalactic extended X-ray emission is the signature of a deep gravitational potential well. Apart from the hypothetical "dark clusters", this potential well coincides with a galaxy overdensity. The system (cluster or group) is therefore detectable using optical information only. In this article, we aim at assessing optical compact structures (massive groups or clusters) corresponding to the X-ray cluster candidates. These systems are expected to manifest themselves as compact structures in redshift space (both spectroscopic and photometric ones) and as localized excess in projected galaxy density maps.

To perform such an analysis, we make use of the two optical data sets mentioned above. The investigated lines of sight (centered on the X-ray emissions) were initially selected if at least two spectroscopic redshift measurements (whatever their values) were available within the X-ray isophotes. The subsequent conditions were more stringent depending on the cluster nature (see below).

The CFHTLS Wide survey and subsequent analyses (e.g. Coupon et al. 2009) provide us with galaxy positions as well as their apparent and absolute magnitudes, photometric redshifts, and the corresponding "galaxy types" $T$ (from the spectral fitting performed during the photometric redshift computation). With the exception of the usual "masking problems" owing to bright stars or CCD defaults, photometric data are homogeneous and allow us to define complete sub-samples in terms of spatial extension or in magnitudes. Limitations to these data are the redshift range within which photometric redshifts are reliable, and the adopted magnitude limit. Here we restrict ourselves to $0.2<z<1.2$ and $i^{\prime}=23$ (see Coupon et al. 2009). This limiting magnitude will partly affect the use of photometric data to detect compact structures. Indeed, the characteristic magnitude $m^{*}$ of the Schechter luminosity function is about $i^{\prime}=20$ at $z=0.5$ and $i^{\prime}=22.5$ at $z=1$, leading to sampled luminosity function ranges of about $m^{*}+3$ to $m^{*}+0.5$ at these respective redshifts. One drawback is therefore that for $z>0.5$ the number of galaxies actually belonging to a structure will be rapidly overcome by the background contamination (see e.g. Table 1 of Adami et al. 2005). One way to fight this contamination will be to use redshift slices defined on a photometric redshift basis (see Mazure et al. 2007), but the range covered in magnitude by structure members will remain limited.

In order not to bias the optical characterization of the X-ray sources, the information concerning the $\mathrm{C} 1, \mathrm{C} 2, \mathrm{C} 3$ classification was used only at the very final stage.

\subsection{Different analysis steps}

The first step concerns the expected compactness in spectroscopic redshift space. To reveal these compact associations, we used the already well tested and used "gap method" (e.g. Biviano et al. 1997; Rizzo et al. 2004). It looks for significant gaps between successive galaxy velocities within the ordered redshift distribution obtained along a given line of sight. As in Adami et al. (2005), we use a gap defined by $g=600(1+z) \mathrm{km} \mathrm{s}^{-1}$, which was optimum for the considered redshift range. When the velocity difference between two successive galaxies is smaller than $g$, they are assigned to belong to a common structure, otherwise they are put in different groups.

Since the lines of sight most of the time sample redshifts up to at least $z=1$, this first step of the analysis ends in general with several groups. Thus, with the mean redshift of every group, a cosmological distance was assigned, a physical region of $500 \mathrm{kpc}$ (radius) defined, and the galaxies within this radius were selected as potential real cluster members. We choose this size as representative of clusters in terms of membership of galaxies w.r.t the field. Taking larger regions would decrease any real contrast, while taking smaller regions would decrease the number of true members. As a second step we then applied the usual ROSTAT tools (Beers et al. 1990) on individual redshift groups to test for final membership and definition of the group properties (robust redshift locations and scales with their corresponding bootstrap errors).

As already mentioned, several groups are in general identified along the lines of sight. Before comparing the galaxy distribution and the X-ray isophotes, we then used when available the CFHTLS photometric redshift information. As a third step, we selected galaxies in photometric redshift slices (of width: $\pm 0.04(1+z)$, see Coupon et al. 2009) around the mean redshift of the considered group and produced iso-contours of numerical galaxy density (see Mazure et al. 2007, for details and previous application). It is expected that the optical group physically associated with the X-ray emission will show up with a clear density contrast located next to the position of the X-ray center. This is because the use of photometric redshift slices removes a large part of the fore and background contaminations. We also looked as another check at the photometric redshift distribution within various central regions compared to the one in the largest available region, conveniently renormalized and defined as the "field". Again, one expects a clear contrast at the redshift values given by the spectroscopy.

An illustration is given with the source XLSSC 013 in the XMM-LSS database. Three main groups were identified along the line of sight $(z \sim 0.2$ with 9 redshifts, $z \sim 0.3$ with 26 redshifts, $z \sim 0.6$ with 5 redshifts). A consecutive examination of both the photometric redshift distribution and the numerical density histograms strongly recommended the $z \sim 0.3$ group to be chosen (see Figs. 2 and 3).

However, as mentioned above, photometric redshift data were not always available and spectroscopic data could be very sparse (our velocity dispersion measurements are then subject to very complex selection functions in the target selection when measuring and collecting galaxy redshifts). The final selection was then done by a visual inspection of X-ray and optical maps taking into account all the informations available. Figure 3 shows the group at $z=0.3$ chosen for XLSSC 013. As an extreme contrary case, we show in Fig. 4 XLSSC 035, for which only a few redshifts were available. The fact that a giant galaxy at $z=0.069$ lies at the center finally pleads in favor of that redshift (Fig. 4) in the present paper. We note however that a $z \sim 0.17$ galaxy layer is also detected along this line of sight and that consequently we could deal with a superposition effect.

\subsection{Results}

We examined $34 \mathrm{C} 1$ candidate X-ray sources. Identification fails for only two lines of sight mainly because very few redshifts were available in the $\mathrm{X}$-ray region and/or we had no photometric redshifts. All identified sources were classified as galaxy clusters; this means that at least $95 \%$ of the $\mathrm{C} 1$ objects are real 

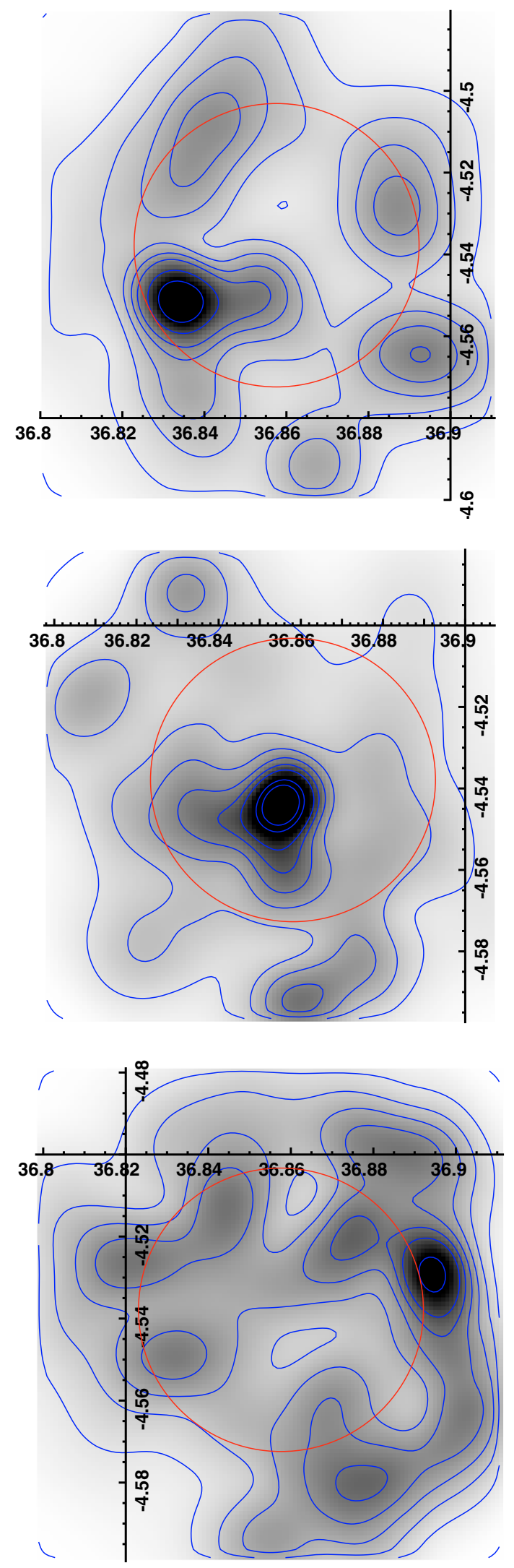

Fig. 2. Isodensity maps of the numerical density of galaxies within photometric slices of width $\pm 0.04(1+z)$ around the group redshifts. From top to bottom: $z \sim 0.2, z \sim 0.3, z \sim 0.6$. The best agreement with the X-ray emission of XLSSC 013 is obtained at $z=0.3$. Large red circles are the same as in Fig. 3.

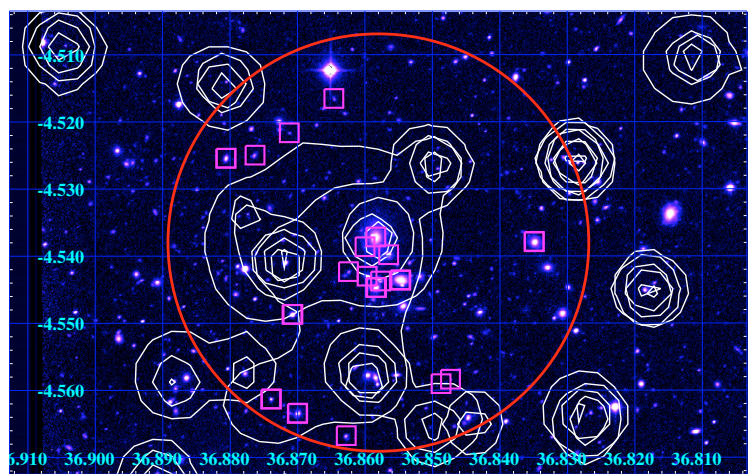

Fig. 3. XMM-LSS X-ray contours for system XLSSC 013 with cluster member galaxies with a measured redshift (between $z=0.3049$ and 0.3112 ) superimposed. The red circle corresponds to a radius of $500 \mathrm{kpc}$ at $z=0.3$.

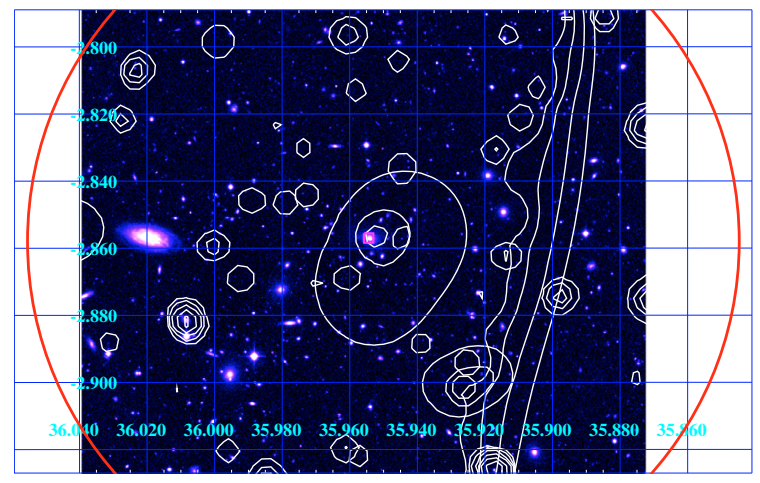

Fig. 4. XMM-LSS X-ray contours for system XLSSC 035 with galaxies with measured redshifts superimposed. The red circle corresponds to a radius of $500 \mathrm{kpc}$ at $z=0.069$.

clusters (when obvious nearby galaxies which also show a diffuse X-ray emission are excluded). Among the C2 and C3 candidates, only those with two redshifts within the X-ray isophotes were selected for the present analysis. As our current spectroscopic data set is heterogeneous and does not provide a systematic targeting of all $\mathrm{C} 2$ and $\mathrm{C} 3$ cluster candidates, it is not possible to draw firm conclusions about the effective contamination rate (in terms of non-cluster sources) for these populations. We can only state that for all C2 (resp. C3) sources with at least two spectroscopic redshifts within the X-ray isophotes, more than $80 \%$ (resp. $50 \%$ ) of the examined sources turned out to be real clusters.

An additional potential X-ray source was also discovered (C555 in Table 4). Not listed in Pierre et al. (2007), this source is merged with XLSSU J022533.8-042540. We detected a very clear associated galaxy compact structure in the optical. A manual extraction of the X-ray source gives a count rate of $0.003 \pm$ 0.001 counts per second ([0.5-2 keV]).

For seven of the analyzed lines of sight, the association between X-ray source and optical galaxy concentration was not obvious or the X-ray source was not significantly different from the background. However, these clusters are identified on the basis of the color-magnitude relation (for two of them) or are detected as significant galaxy overdensities in Adami et al. (2010) using photometric redshifts during the analysis. All these objects have been classified as $\mathrm{C} 0$ clusters.

The $\mathrm{C} 1, \mathrm{C} 2, \mathrm{C} 3$, and $\mathrm{C} 0$ clusters are presented in Tables 2-5. Almost two thirds have been confirmed with 


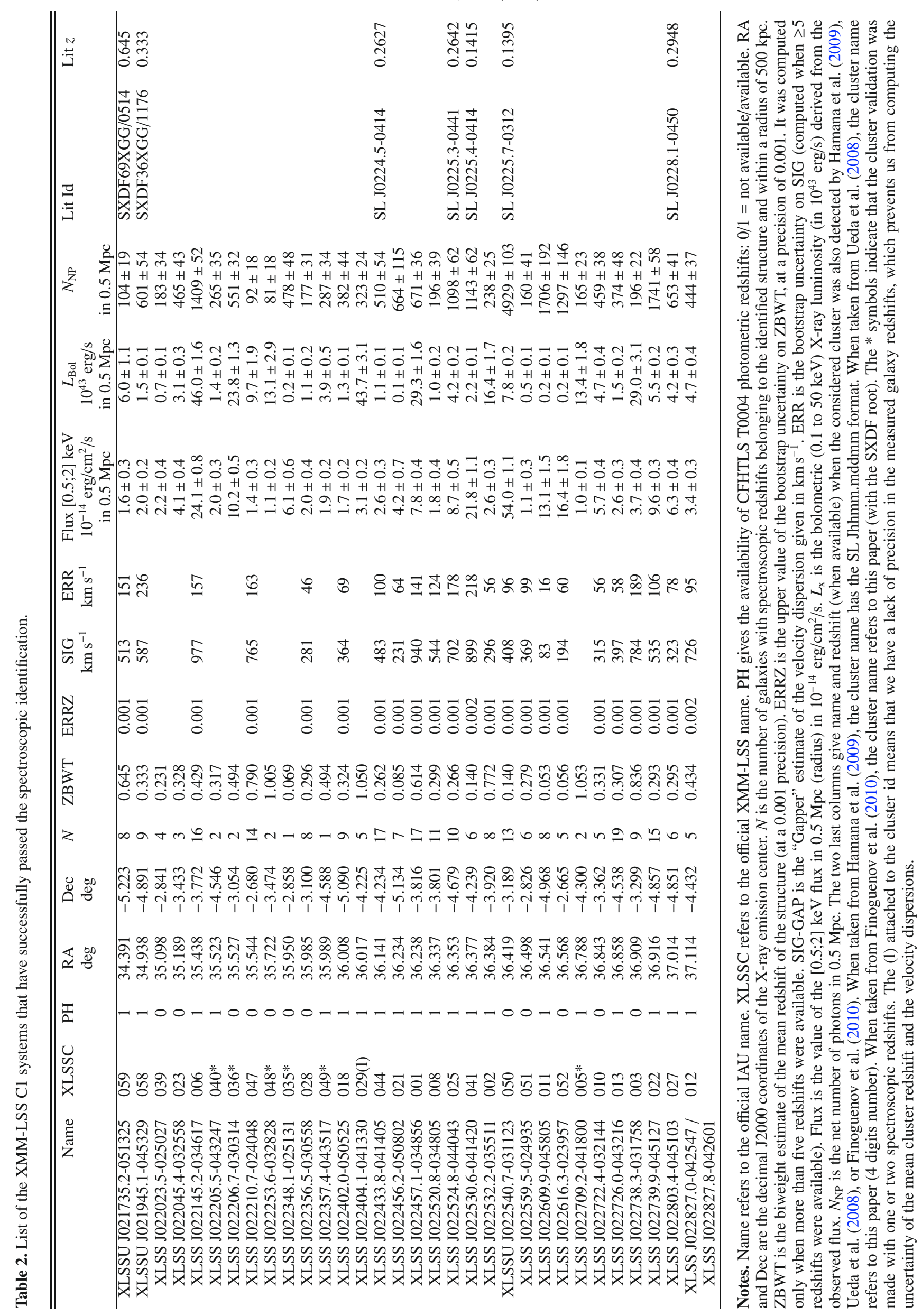


C. Adami et al.: Optical assessment and comparative study of the $\mathrm{C} 1, \mathrm{C} 2$, and $\mathrm{C} 3$ cluster classes

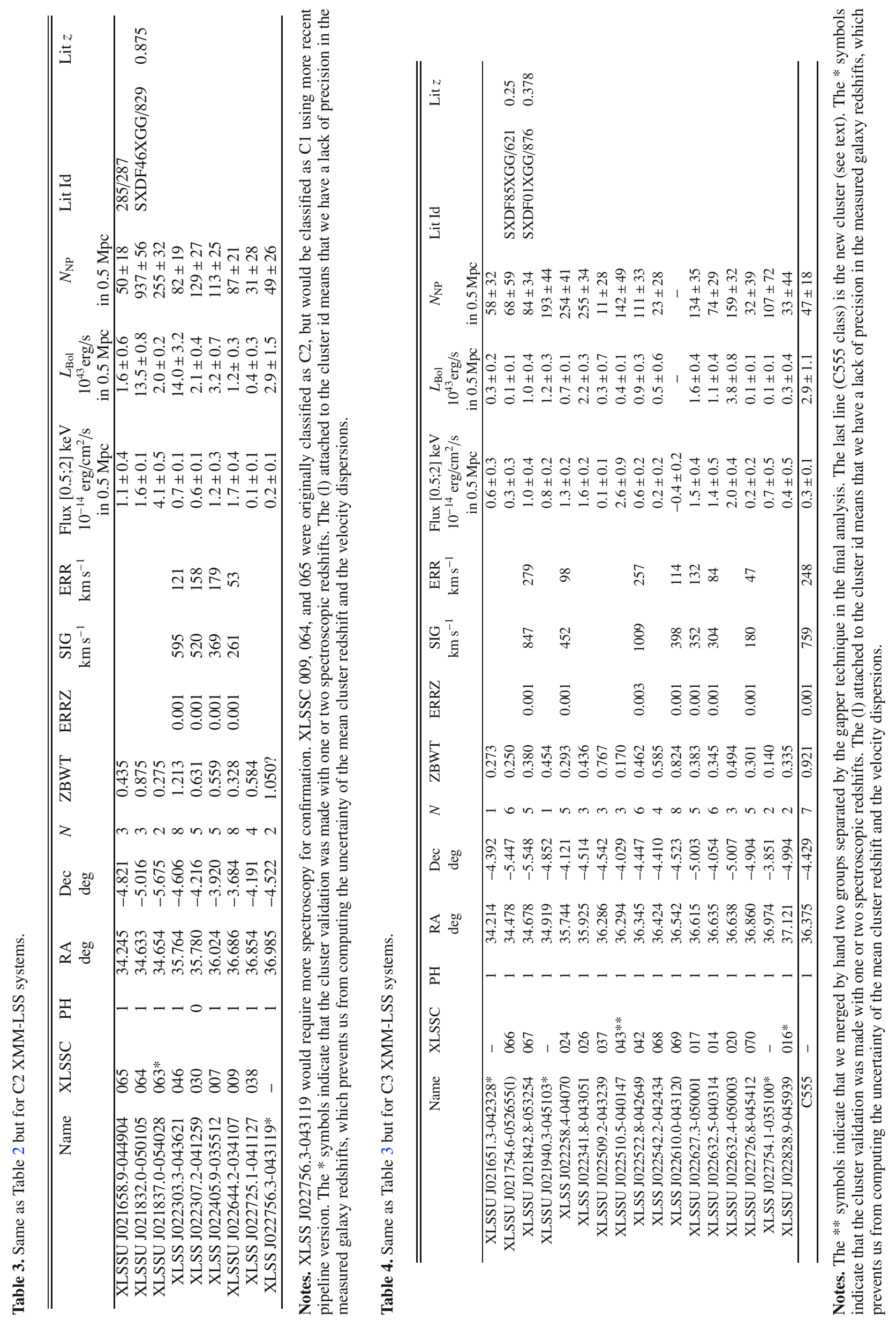


Table 5. Same as Table 3 but for $\mathrm{C} 0$ clusters.

\begin{tabular}{cccccccccc}
\hline \hline Name & XLSSC & PH & $\begin{array}{c}\text { RA } \\
\text { deg }\end{array}$ & $\begin{array}{c}\text { Dec } \\
\text { deg }\end{array}$ & $N$ & ZBWT & ERRZ & $\begin{array}{c}\text { SIG } \\
\mathrm{km} \mathrm{s}^{-1}\end{array}$ & $\begin{array}{c}\text { ERR } \\
\mathrm{km} \mathrm{s}^{-1}\end{array}$ \\
\hline $022207.9-042808^{*}$ & - & 1 & 35.533 & -4.469 & 2 & 0.316 & & & \\
$022402.4-051753$ & 000 & 1 & 36.010 & -5.298 & 11 & 0.496 & 0.001 & 435 & 88 \\
$022405.0-041612$ & - & 1 & 36.021 & -4.270 & 8 & 0.862 & 0.001 & 457 & 70 \\
$022528.3-041536$ & 045 & 1 & 36.369 & -4.261 & 4 & 0.556 & & & \\
$022550.4-044500^{*}$ & - & 1 & 36.460 & -4.750 & 2 & 1.529 & & & \\
$022647.5-041428 *$ & - & 1 & 36.698 & -4.241 & 1 & 0.742 & & & \\
$022829.7-031257^{*}$ & - & 0 & 37.124 & -3.216 & 2 & 0.313 & & & \\
\hline
\end{tabular}

Notes. An approximate upper limit for the X-ray luminosity of these clusters would be the faintest detected value for $\mathrm{C} 3 \mathrm{clusters:} \sim 0.08 \times 10^{43} \mathrm{erg} / \mathrm{s}$.

dedicated spectroscopy only and $10 \%$ have been confirmed with dedicated spectroscopy supplemented by literature redshifts.

We compared the cluster redshifts listed in the present paper (see also next section) with the estimates already published within the XMM-LSS framework (from Pacaud et al. 2007; and Bremer et al. 2006: 29 C1 clusters and 1 C2 cluster), and we found the expected good agreement. This is not surprising as Pacaud et al. (2007) and Bremer et al. (2006) are included in our spectroscopic redshift sample. However, the redshift measurements were repeated on a more homogeneous basis and sometimes with new data. The difference is only $0.00075 \pm 0.00329$ when excluding XLSSC 035. For this cluster, we detected a possible error in the individual redshifts measurement process. The central galaxy seems to be at $z=0.069$ and not 0.17 as stated in Pacaud et al. (2007: the cluster redshift changed to $z=0.069$ ). We are in the process of acquiring more data in order to definitively solve this case. We also note that the central galaxies of XLSSC 028 are also at $z \sim 0.3$ and not at $z \sim 0.08$ as stated in Pacaud et al. (2007: cluster redshift unchanged at $z \sim 0.3$ ).

The agreement is very good for the $[0.5-2 \mathrm{keV}]$ fluxes measured in a $500 \mathrm{kpc}$ radius (Fig. 5).

For the Subaru Deep Survey region, we compared our detections with the extended X-ray source catalog of Ueda et al. (2008) and with the compact structure catalog of Finoguenov et al. (2010). Nine of our X-ray clusters are inside the area covered by these catalogs and six are also detected by these authors. Redshifts always agree well. Finoguenov et al. (2010) list in their paper 57 compact structures inside this area. However, their selection function (completeness/contamination) for the $\mathrm{X}$-ray extended sources as well as the characteristics of these sources (extent-measurement along with error or likelihood) are not fully published (see however Finoguenov et al. 2009), thus preventing any meaningful comparison between the two samples. Moreover, as shown by Pacaud et al. (2006), if a flux limit is not set very high, it cannot define a complete uncontaminated sample of extended sources.

We finally performed a comparison with independently optically detected clusters in the literature. Limiting ourselves to studies that give a galaxy velocity dispersion estimate, we have five detections in common with Hamana et al. (2009: see Table 2). All these clusters are C1 structures. Redshifts always agree well. Galaxy velocity dispersions are also consistent within error bars with an exception for XLSSC 050, where we find $408 \pm 96 \mathrm{~km} \mathrm{~s}^{-1}$ and where Hamana et al. (2009) find $739_{-86}^{+150} \mathrm{~km} \mathrm{~s}^{-1}$. Because this structure is very complex, the galaxy velocity dispersion is very dependent on the selected galaxies and on the exact center choice.

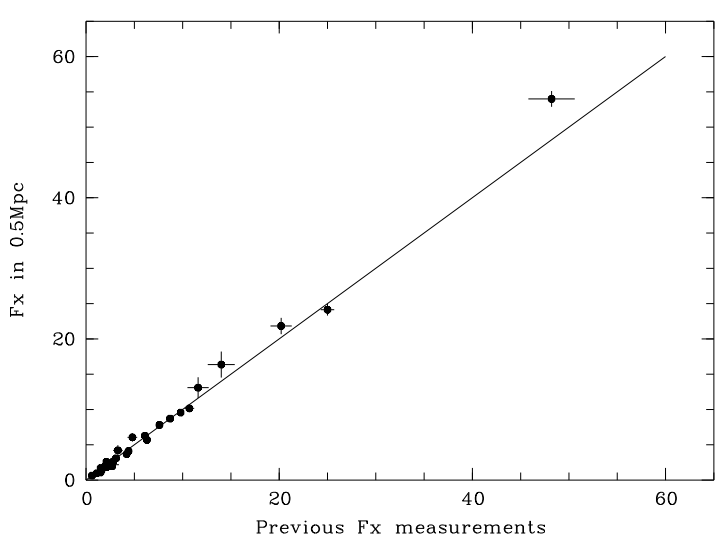

Fig. 5. Previous and present cluster flux (in a $0.5 \mathrm{Mpc}$ radius) comparisons.

\subsection{Updated X-ray luminosities}

We apply the principle of "aperture photometry" to the flux measurement of the X-ray clusters, which avoids any other assumption than spherical symmetry for the cluster shape. We note that Pacaud et al. (2007) used a beta-model fitting, which is not possible for the larger sample presented here, which comprises faint objects. For these C2 and C3 objects with sometimes only some hundred counts, it is not possible to perform a semi-interactive spatial fit as in Pacaud et al. (2007), i.e. letting the core radius and the beta value as free parameters. The resulting uncertainty would be very large.

We integrate the count rate in concentric annuli and derive the uncertainties by using the Poisson statistic. Then, considering the count rate in each annulus, we stop the integration at the radius of the annulus for which the corresponding countrate increase is comparable to the background 1-sigma fluctuation. This program operates in semi-interactive mode, leaving the possibility to optimize the determination of the X-ray centroid and of the background level. The measurement yields the total MOS $1+$ MOS2 + PN count-rate within a radius $500 \mathrm{kpc}$ for each cluster. The fluxes were obtained assuming a fixed conversion factor into the [0.5-2] $\mathrm{keV}$ band using a constant conversion factor of $9 \times 10^{-13}\left[\left(\mathrm{erg} / \mathrm{cm}^{2} / \mathrm{s}\right) /(\mathrm{cnts} / \mathrm{s})\right]$. This value was calculated using Xspec from an APEC emission model with the following parameters: $z=0.5, T=2 \mathrm{keV}, \mathrm{Nh}=2.6 \times 10^{20} \mathrm{~cm}^{-2}$, $\mathrm{Ab}=0.3$. Bolometric luminosities (also within a $500 \mathrm{kpc}$ radius) listed in the tables were also calculated with Xspec from the measured fluxes using the Pacaud et al. (2007) and the Bremer et al. (2006) temperatures when available. We used the redshifts 


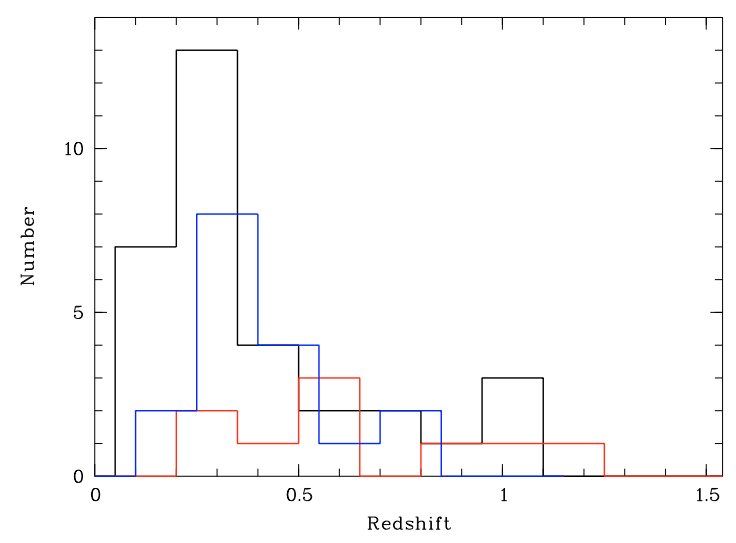

Fig. 6. Redshift distribution for the three classes C1 (black histogram), C2 (red histogram), C3 (blue histogram).

described below. For clusters not listed in these papers (probably low mass structures), we used $T=1.5 \mathrm{keV}$.

\section{Global properties of the various classes}

We will consider only clusters that are successfully identified from here until the end of the paper.

\subsection{Rich and poor compact structures}

For X-ray sources unambiguously identified with optical velocity structures, one has to address the question: has the $\mathrm{C} 1, \mathrm{C} 2, \mathrm{C} 3$ classification a physical basis, or is it only reflecting the X-ray selection process?

As a first step, we look at the redshift distribution of the cluster $\mathrm{C} 1, \mathrm{C} 2$, and $\mathrm{C} 3$ classes (Fig. 6). For the $32 \mathrm{C} 1$, the $9 \mathrm{C} 2$, and the $17 \mathrm{C} 3$ the mean redshift is $0.41,0.66$, and 0.38 .

Comparing the $\mathrm{C} 1$ and $\mathrm{C} 3$ distributions and their almost similar mean redshifts and ignoring the $z \geq 0.5 \mathrm{C} 3$ structures for a while, it is tempting to consider $\mathrm{C} 1$ to be in the most cases "X-ray bright and optical nearby $(z \leq 0.4)$ rich systems" and most of the $\mathrm{C} 3$ as "faint and poor" at $z \sim 0.4$ redshift. The more distant $\mathrm{C} 3$ clusters would be somewhat distant $\mathrm{C} 1$-like and therefore "rich". C2 clusters would be a mix of nearby poor and distant rich clusters.

We can define alternative categories to the $\mathrm{C} 1, \mathrm{C} 2, \mathrm{C} 3$ classification. For instance, we chose to group the clusters as a function of their X-ray luminosity. Clusters more luminous than $10^{44} \mathrm{erg} / \mathrm{s}$ were called the X-ray most luminous sample. Clusters between $10^{43}$ and $10^{44} \mathrm{erg} / \mathrm{s}$ were called the X-ray luminous sample. Clusters below $10^{43} \mathrm{erg} / \mathrm{s}$ were called the $\mathrm{X}$-ray moderately luminous sample. Finally, clusters without any $\mathrm{X}$-ray detection ( $\mathrm{C} 0$ clusters) were considered separately. We give in Fig. 7 the redshift distribution of these four categories. As expected because of the relatively small angular coverage of the XMM-LSS survey, the most luminous clusters are mainly distant objects. Similarly, moderately luminous clusters are quite nearby objects because our X-ray selection function does not allow us to detect them when they are distant, according to the well known Malmquist bias.

We show in Fig. 8 a synthethic view of the clusters listed in Tables 2-4, allowing the reader to visualize the different classes (C1, C2, C3, most luminous, luminous, and moderately luminous) in a redshift versus X-ray luminosity diagram.

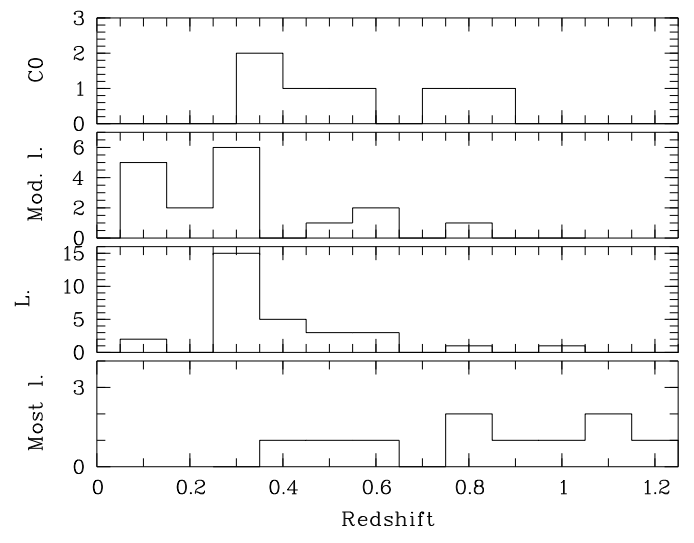

Fig. 7. Redshift distribution for the most luminous (Most 1.), luminous (L.), moderately luminous (Mod. 1.), and C0 clusters.

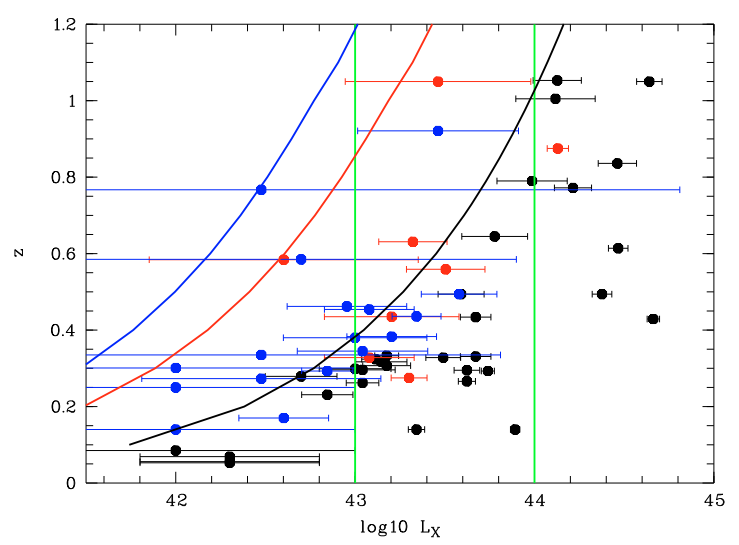

Fig. 8. Present paper cluster distribution in a $\log 10\left(L_{X}\right)$ versus redshift diagram. The two vertical green lines separate the most luminous, luminous, and moderately luminous clusters. Black disks are $\mathrm{C} 1$ clusters, red disks are $\mathrm{C} 2$ clusters, blue disks are $\mathrm{C} 3$ clusters. We also show as black, red, and blue curves the detection limit of the lowest X-ray flux cluster in $\mathrm{C} 1, \mathrm{C} 2$, and $\mathrm{C} 3$ classes.

\subsection{Optical richness}

We know (e.g. Edge \& Stewart 1991) that optical and X-ray cluster properties should be relatively well correlated. It is then necessary to characterize the optical richness $\left(N_{\text {Rich }}\right)$ of our clusters. This is done by taking first the number of galaxies in the region of $500 \mathrm{kpc}$ (radius), within the photometric redshift slice zmean $\pm 0.04(1+z)$ and with magnitude less than $m^{*}+3$. That number is then corrected by the "field contribution" estimated in the same manner within $1 \mathrm{Mpc}$ to give the final estimate. This richness value is probably not accurate enough in terms of absolute value, but can be used in a relative way when comparing a structure to another one. We also note that given the CFHTLS wide magnitude limit, we adopted $\left(i^{\prime}=23\right)$, only $z \leq 0.5$ clusters are sampled deeply enough to reach $m^{*}+3$. We therefore only considered these clusters in order to avoid to have biased optical richnesses.

Fitting a richness-velocity dispersion for all $z \leq 0.5$ compact structures for which both data were available, we get:

$\log (\sigma)=(0.45 \pm 0.24) \log \left(N_{\text {Rich }}\right)+(1.96 \pm 0.38)$.

This is compatible within the uncertainties with the value of Yee \& Ellingson (2003) for similar data:

$\log (\sigma)=(0.55 \pm 0.09) \log \left(B_{\mathrm{cg}}\right)+(1.26 \pm 0.30)$. 


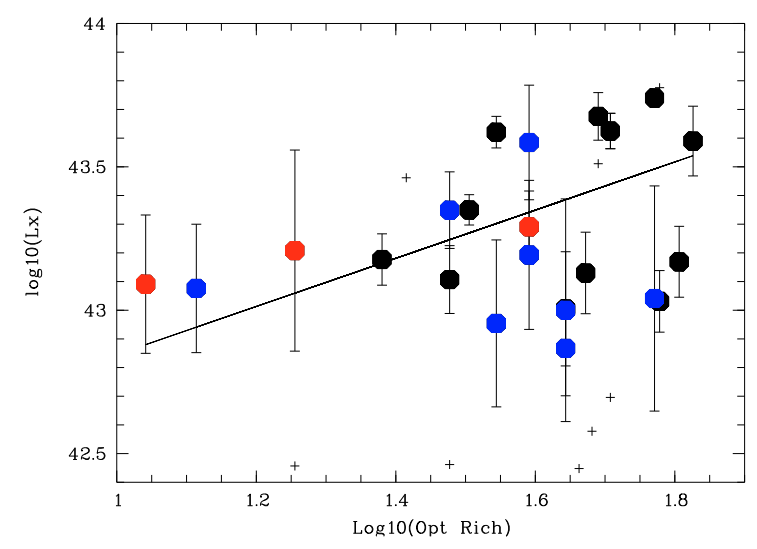

Fig. 9. $\log \left(N_{\text {Rich }}\right)$ versus $\log \left(L_{\mathrm{X}}\right)$. Crosses are clusters at signal-to-noise lower than 2 regarding the $\mathrm{X}$-ray luminosity. Disks are clusters at signalto-noise greater than 2 and at $z \leq 0.5$ (black: $\mathrm{C} 1$, red: $\mathrm{C} 2$, blue: $\mathrm{C} 3$ ). They give the following fit: $\log \left(N_{\text {Rich }}\right)=(0.84 \pm 0.51) \log \left(L_{X}\right)+(7.2 \pm$ $0.81)$.

We now test richness and velocity dispersions versus X-ray properties. We first consider $z \leq 0.5$ compact structures with known $\mathrm{X}$-ray luminosity and optical richness. We selected only C1, C2 and C3 clusters with X-ray luminosity at least two times higher than the associated uncertainty. We show in Fig. 9 the possible relation between the logarithm of $N_{\text {Rich }}$ and of $L_{\mathrm{x}}$. The linear regression between the two parameters has a slope of $0.84 \pm 0.51$. We note that this value only appears slightly significantly different from a null slope.

There is a single clear interloper: XLSSC 006 at $z \sim 0.43$ (outside of the box shown in Fig. 9). This is one of the most massive clusters in our sample. The observed spectra in the cluster center do not show any sign of AGN activity, so we have no reason to believe that the X-ray flux is polluted by a point source. This cluster shows signs of major substructures in the velocity distribution, and this may explain its relatively high $L_{\mathrm{x}}$ value compared to its optical richness. Resulting compression in the intracluster medium could increase the gas density, resulting in an enhanced X-ray luminosity.

Considering now clusters at $z \leq 0.5$ with a known $X$-ray temperature (from Pacaud et al. 2007) and a measured galaxy velocity dispersion, we searched for a relation between $N_{\text {Rich }}$, velocity dispersion, and X-ray temperature. Figure 10 shows the relation between $\log \left(N_{\text {Rich }} \sigma^{2}\right)$ and $\log \left(T_{\mathrm{X}}\right)$. We expect a linear relation because $\left(N_{\mathrm{Rich}} \sigma^{2}\right)$ is at least a qualitative measurement of the kinetic energy of the clusters, therefore close to the X-ray temperature. Error bars on $\left(N_{\text {Rich }} \sigma^{2}\right)$ are $68 \%$ uncertainties and are computed assuming a perfect knowledge of the richness and the error bars on $\sigma^{2}$ given in Tables 2-4. As quoted in Table 2, these uncertainties are computed with a bootstrap technique.

We have two outliers: XLSSC 027 and XLSSC 018. The source XLSSC 027 is known to have strong discrepancies between galaxy and weak lensing equivalent velocity dispersions $\left(898_{-527}^{+523} \mathrm{~km} \mathrm{~s}^{-1}\right.$ from Gavazzi \& Soucail (2007) against $323 \pm 78 \mathrm{~km} \mathrm{~s}^{-1}$ for our own galaxy velocity dispersion and $447_{-52}^{+82} \mathrm{~km} \mathrm{~s}^{-1}$ for the Hamana et al. (2009) galaxy velocity dispersion). We note that using the weak lensing equivalent velocity dispersion puts XLSSC 027 close to the best-fit relation. We also note that this cluster has close contaminants at $z=0.31$ and 0.38 detected along the line of sight. This could also affect the measurement of the optical richness via the background estimate.

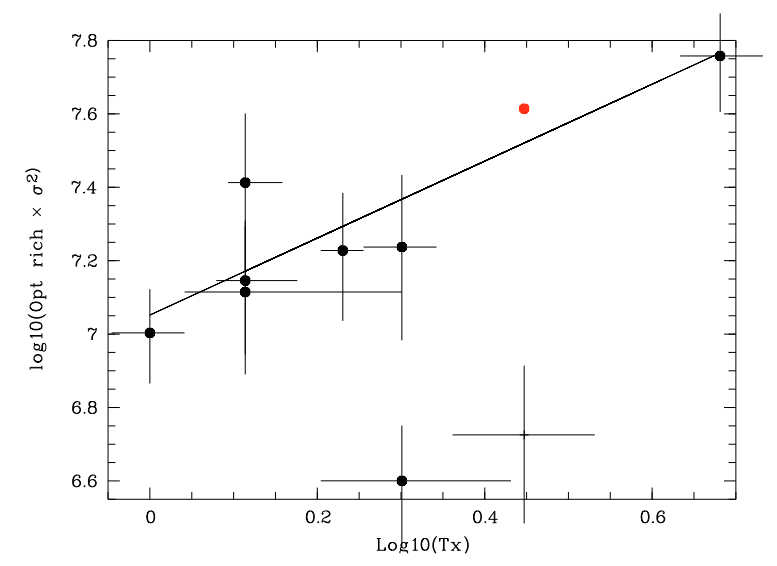

Fig. 10. $\log \left(N_{\text {Rich }} \sigma^{2}\right)$ versus $\log \left(T_{\mathrm{X}}\right)$ for the $z \geq 0.5$ clusters (all $\mathrm{C} 1$ but XLSSC 046 which is C2). The + sign indicates XLSSC 027 and translates to the red disk when replacing the galaxy velocity dispersion by the weak lensing estimate from Gavazzi \& Soucail (2007). The continuous line is the fit (computed without XLSSC 027 and XLSSC 018): $\log \left(N_{\text {Rich }} \sigma^{2}\right)=(1.05 \pm 0.13) \log \left(T_{\mathrm{X}}\right)+(7.05 \pm 0.07)$.

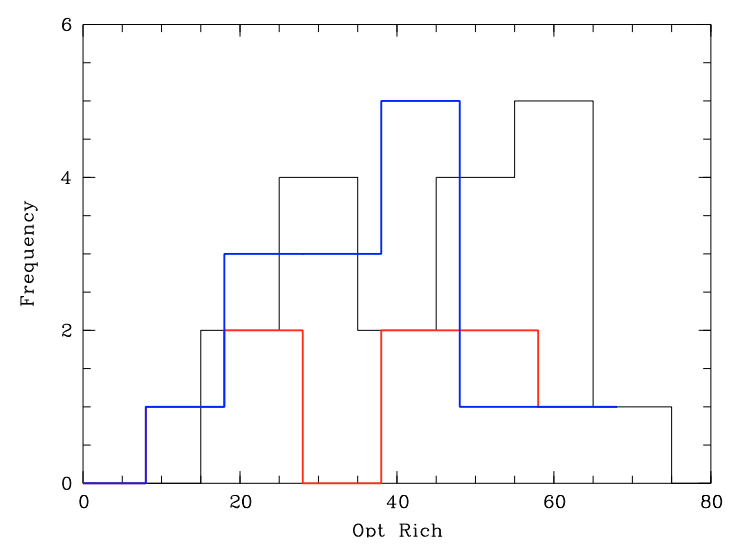

Fig. 11. Distribution of the optical richness for the three classes $\mathrm{C} 1$ (thin black), C2 (thick red), and C3 (thick blue).

XLSSC 018 (without any sign of major substructures: see below) would need a higher optical richess and/or a higher galaxy velocity dispersion, or a lower X-ray temperature to fall in the best-fit relation. The last solution in unlikely because only an X-ray temperature on the order of $0.4 \mathrm{keV}$ would place XLSSC 018 on the best-fit relation. A possible explanation would be that we are dealing with a structure close to a fossil group (even if it does not satisfy the characteristics of this class of compact structures). A significant part of the cluster member galaxies could have merged with the central galaxy, then depopulating the $\leq m^{*}+3$ mag range and diminishing the measured optical richness.

In conclusion, and despite a few detected interlopers, the general agreements show the statistical reliability of our optical richness and galaxy velocity dispersion estimates.

Figure 11 shows the histograms of the richness for the three classes. C1 has a mean $N_{\text {Rich }}$ of $45, \mathrm{C} 2$ a mean $N_{\text {Rich }}$ of 37 and C3 a median $N_{\text {Rich }}$ of 35 .

\subsection{Substructure level in velocity space}

Our spectroscopic catalogs are generally too sparse to allow precise substructure analyses. However, limiting ourselves to the confirmed clusters with available CFHTLS data and with more 
than nine redshifts in the structure (10 clusters: XLSSC 013, XLSSC 025, XLSSC 022, XLSSC 006, XLSSC 008, XLSSC 001, XLSSC 000, XLSSC 018, XLSSC 044, and XLSSC 058), we applied the Serna-Gerbal method (Serna \& Gerbal 1996: SG hereafter) to these spectroscopic catalogs. Two of them (XLSSC 006 and XLSSC 001) are from the most luminous cluster category. All the others except for XLSSC 000 (which is a C0 cluster) are members of the luminous cluster category. The SG method is widely used to characterize the substructure level in clusters of galaxies (e.g. Adami et al. 2009). Basically, the method allows galaxy subgroups to be extracted from a catalog containing positions, magnitudes, and redshift, based on the calculation of their relative binding energies. The output of the SG method is a list of galaxies belonging to each group, as well as information on the binding energy and mass estimate of the galaxy structures.

Because the spectroscopic catalogs are still relatively sparse, we will only be able to detect very prominent substructures, but this is a good way to check for example if the analyzed clusters are in the process of a major merging event.

Of the 10 analyzed clusters, only two (which both belong to the most luminous cluster category) present signs of substructures (XLSSC 006 with two dominant galaxies in its center and XLSSC 001) with two detected sub-groups. We checked if these two clusters were atypically sampled in terms of number of available redshifts. XLSSC 001 has 17 redshifts and XLSSC 006 16 redshifts. Three other clusters without detected signs of substructures are as well sampled: XLSSC 013 has 19 redshifts, XLSSC 022 has 15 redshifts, and XLSSC 044 has 17 redshifts. The substructure detection therefore does not seem to be entirely due to selection effects depending on the available number of redshifts. As a conclusion, all tested most luminous clusters show signs of substructures, while none of the other tested clusters show similar signs. This would agree well with a scenario where the most massive clusters are regularly fed by their surrounding large-scale structure in terms of infalling groups. Less luminous clusters would already be close to their equilibrium, with a less intense infalling activity. This has to be confirmed with larger spectroscopic samples, however.

\subsection{Relation between XMM-LSS clusters and their parent cosmic web portion}

The previous subsection naturally raises the question of the characteristics of cosmological surrounding filaments. Numerical simulations place clusters of galaxies at the nodes of the cosmic web. Clusters are then growing via accretion of matter flowing along the cosmic filaments. This unquestionable scenario for massive clusters is less evident for low-mass compact structures as groups. These groups could also form along the cosmic filaments as suggested for example for fossil groups by Adami et al. (2007a). Moreover, even for the most massive compact structures, the precise process of filament matter accretion is only assessed most of the time by individual cluster studies (e.g. Boué et al. 2008). The XMM-LSS cluster sample presented in this paper offers a unique opportunity to investigate the cluster-filament connection with a well controled sample.

\subsubsection{General counting method}

We first have to detect the filaments connected to a given cluster. These filaments have a very low mass and are young dynamical structures. It is therefore very difficult to detect them through X-ray observations. This is possible only in a few peculiar cases (e.g. Boué et al. 2008; Werner et al. 2008) and with very long integration times. The XMM-LSS exposure times are anyway not well suited to such detections. We therefore used optical CFHTLS photometric redshift catalogs.

- We first selected only clusters at $z \leq 0.5$ in order to be able to sample the galaxy population deeply enough to potentially detect the filaments, given the $i^{\prime}=23$ magnitude limit for the photometric redshift catalog as demonstrated in Adami et al. (2010);

- for a given cluster, we selected galaxies with photometric redshifts in a $0.04 \times(1+z)$ slice around the cluster redshift;

- we then computed the number of galaxies in the slice in 72 angular sectors 10 degrees wide each, with position angles between 0 and 360 degrees. Each sector was overlapping the previous one by 5 degrees. We did this exercise for galaxies in a circle of $2.5 \mathrm{Mpc}$ radius, and in an annulus between 2.5 and $5 \mathrm{Mpc}$.

\subsubsection{Filament detection and signal enhancement}

Intuitively, if a given sector is significantly more populated than other sectors, it means that this sector includes a galaxy overdensity which could be explained by a filament or by a group along a filament. The question is then to define a significance level. For a given cluster (and then a given redshift slice) and a given radius, we chose to compute the mean and dispersion of the galaxy numbers in the 72 considered sectors. If a given sector had a number of galaxies larger than the mean +3 times the dispersion, we considered this sector as hosting a potential cosmic filament portion.

However, individual clusters exhibit at best a single 3- $\sigma$ significant candidate filament. This is because of the intrinsic very low galaxy density in filaments. Moreover, the goal of the present section is not to make individual cluster studies, but to draw statistical tendencies. In order to enhance the significance of the filament detections, we therefore stacked different clusters, considering two categories: the luminous and the moderately luminous clusters. Other categories did not have enough cluster members in the selected redshift range. This technique is based on the assumption that the angular separation between different filaments feeding a given cluster is more or less constant. In order to make such a stack we now need to homogeneize the cluster position angles. We chose the position angle of the highest galaxy overdensity (the PA), limiting ourselves to clusters with a detection more significant than the 3- $\sigma$ level. Selected clusters were rotated to have their most significant filament at an arbitrary position angle of 180 degrees (east-west direction). In order to check if this alignment technique has a physical meaning, we superposed in the same way the X-ray images using the position angles defined by the highest galaxy overdensities (more significant than the 3- $\sigma$ level). After rotating these X-ray images, we spatially rescaled them to physical units (kpc) according to redshift, and we simply summed them up, taking into account the corresponding weight maps. The resulting point spread function is a mean of the individual values and remains small compared to cluster typical sizes. Figure 12 shows that we generate in this way a clearly elongated synthethic X-ray cluster along the $180 \mathrm{deg}$ direction. The measured ellipticity of the external isophote is equal to 0.41 . If instead of a simple sum we compute the median of the images (Fig. 12), the resulting ellipticity of the external isophote is still 0.36 . Finally, if we combine the X-ray images without correcting by the optically determined 


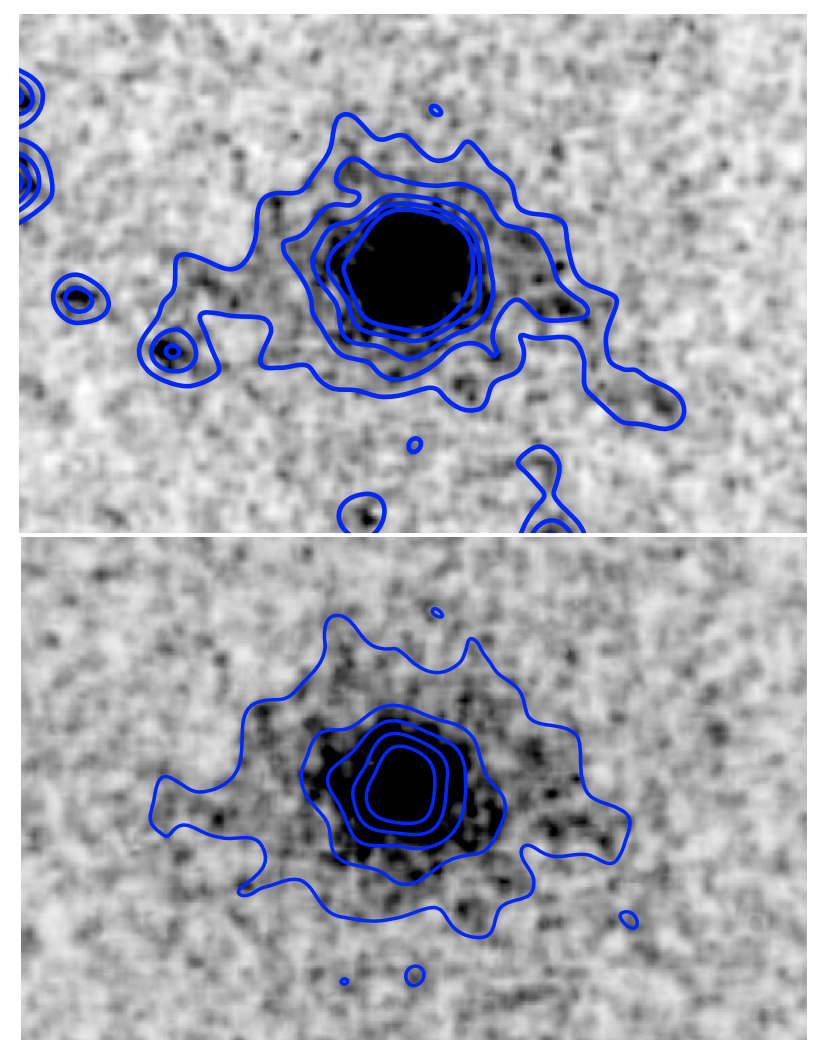

Fig. 12. Stacked X-ray images with position angles defined by the highest galaxy overdensities aligned along the 180 deg arbitary angle. Images were rescaled to physical units according to cluster redshift. Image size is $1 \mathrm{Mpc} \times 650 \mathrm{kpc}$. Contours were drawn with a $20 \times 20$ pixel smoothing. Upper figure: mean stacking. Lower figure: median stacking.

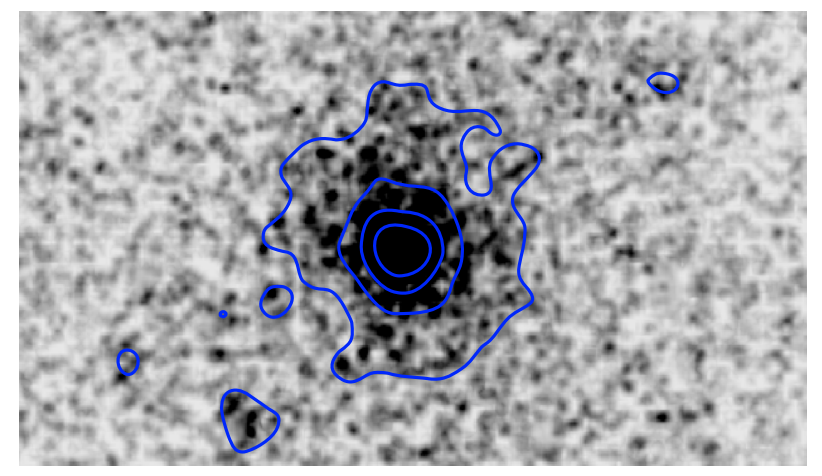

Fig. 13. Same as Fig. 12 with a median stacking and without any position angle correction.

orientation, we produce Fig. 13, which shows a basically null ellipticity.

If the galaxy-defined prefered orientations are valid, the detected elongation in X-rays is an expected behavior because $\mathrm{X}$-ray emiting groups are also expected to fall onto clusters coming from surrounding filaments (see e.g. Boué et al. 2008).

We have to take into account the cluster redshift before merging their galaxy populations. A single catalog magnitude limit would evidently increase the weight of nearby clusters compared to more distant ones. We therefore limited the galaxy catalogs to $i^{\prime}=23$ at $z=0.5$. The limits were brighter by $D$ magnitudes for nearer clusters, with $D$ being the distance moduli difference between the cluster redshifts and $z=0.5$.

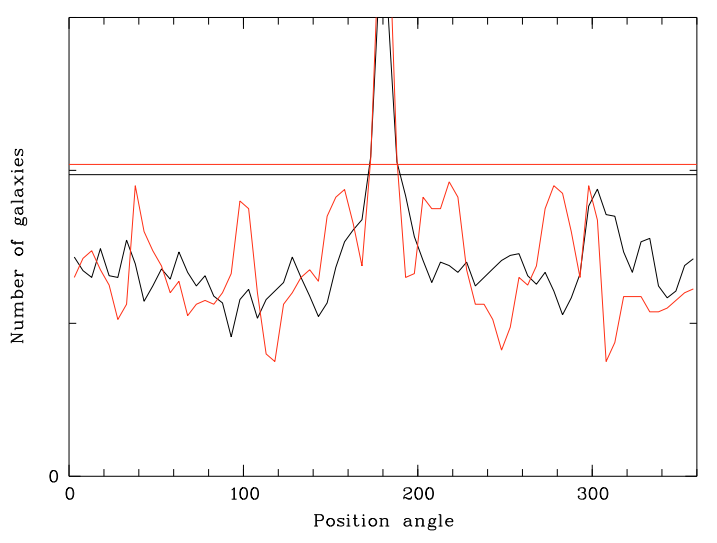

Fig. 14. Stacked angular galaxy counts (in arbitray units) for luminous (black line) and moderately luminous (red line) clusters in annuli of $[2.5,5] \mathrm{Mpc}$. The horizontal lines show the 3- $\sigma$ detection levels.

Renormalizing finally the galaxy counts by the number of selected clusters in a given class, we are able to produce figures that give the mean galaxy counts as a function of the angular position.

\subsubsection{Results}

We first draw stacked (using the previously defined PA of each cluster) angular galaxy counts for luminous and moderately luminous clusters in the annulus [2.5,5] Mpc (Fig. 14). The minimal and maximal radii have been choosen to be close to the mean virial radius of clusters (e.g. Carlberg et al. 1996) and not too large in order to limit the contamination by other clusters. These annuli will therefore mainly sample the infalling galaxy layers, just before the cluster-dominated areas. As expected, the signal from the most significant filament candidate is drastically increased, but no other features are detected at the 3- $\sigma$ level besides the main filament.

We repeat now the same exercise inside a $2.5 \mathrm{Mpc}$ radius central area (Fig. 15). This area is mainly dominated by the clusters themselves (the few hundreds of kpc central areas) and by the galaxy layers just beginning to experience the cluster influence (close to the virial radius). We therefore investigate the cluster region as fed by the connected filaments. The signal from the main filaments is still increased. Other significant filament candidates are detected at the 3- $\sigma$ level mainly for the moderately luminous cluster sample.

This difference between the $2.5 \mathrm{Mpc}$ radius central area and the $[2.5,5] \mathrm{Mpc}$ annulus could be explained if the immediate vicinity of the considered clusters would be depopulated by the potential well of the clusters, diminishing the contrast between cosmic filaments and voids. Larger spectroscopic redshift samples will soon become available in the area and will allow us to refine our results in future works.

\section{Cluster galaxy populations characteristics}

We now investigate the optical properties of the galaxy populations in association with the X-ray clusters. We refer the reader to Urquhart et al. (2010) for individual studies of the clusters providing a temperature measurement. 


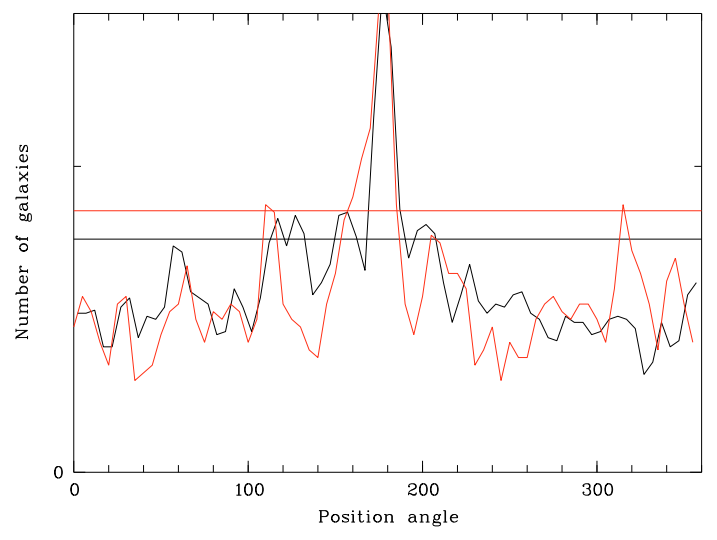

Fig. 15. Stacked angular galaxy counts (in arbitray units) for luminous (black line) and moderately luminous (red line) clusters inside a circle of $2.5 \mathrm{Mpc}$ radius. The horizontal lines show the $3-\sigma$ detection levels.

\subsection{Rest frame red sequences}

The so-called red sequence (RS hereafter) commonly shows up to at least $z \sim 1.2$ (e.g. Stanford et al. 2002) in the massive structures. It is also detected in a less compact state in field galaxy populations up to $z \sim 2$ (Franzetti et al. 2007). We therefore searched for RSs in our sample of clusters. This sample does not provide enough statistics per cluster to perform individual studies. The optimal strategy is therefore to build synthetic clusters by gathering galaxies for clusters of the same category. We therefore considered four classes of clusters: the most luminous, the luminous, the moderately luminous, and the $\mathrm{C} 0$ clusters. Because the RS is a powerful tool to characterize the evolutionary stage of the cluster galaxy populations (e.g. Adami et al. $2007 \mathrm{~b}$ ), such a study will allow us to assess the properties of these four cluster classes.

In order to be able to stack different clusters at different redshifts, rest frame absolute magnitudes were computed in the process of getting photometric redshifts with LePhare (e.g. Ilbert et al. 2006) and we used these magnitudes to compute colors. Basically the method consists in selecting the observed band that is the closest of the requested rest frame band to compute the magnitude in this band by applying correction factors. They are described in the annex of Ilbert et al. (2005), including for example k-correction. This method is the closest of the observations and minimizes our dependence on the assumed spectral energy distributions, which could not be exactly the same in clusters and in the field (see also annex I of the present paper).

\subsubsection{Red sequence using spectrocopic redshifts}

In a first step we look only at galaxy members with spectroscopic redshifts rather than photometric ones to remove potential interloper galaxies that are non cluster members but close to the cluster redshift. These galaxies could be interpreted as cluster members considering only photometric redshifts because of their limited precision. We here consider $u^{*}-r^{\prime}$ rest frame colors and look at their behavior versus rest frame $r^{\prime}$ absolute magnitude.

Figure 16 shows that a RS is present with $u^{*}-r^{\prime} \sim 2$ for all clusters. The slopes of the RS appear to agree well with literature estimates (e.g. Adami et al. 2007b: between -0.1 and -0.02 for the Coma cluster) and are given in Table 6. As expected, RSs are populated by early-type galaxies, while later-type objects are grouped in a much less compact bluer sequence.
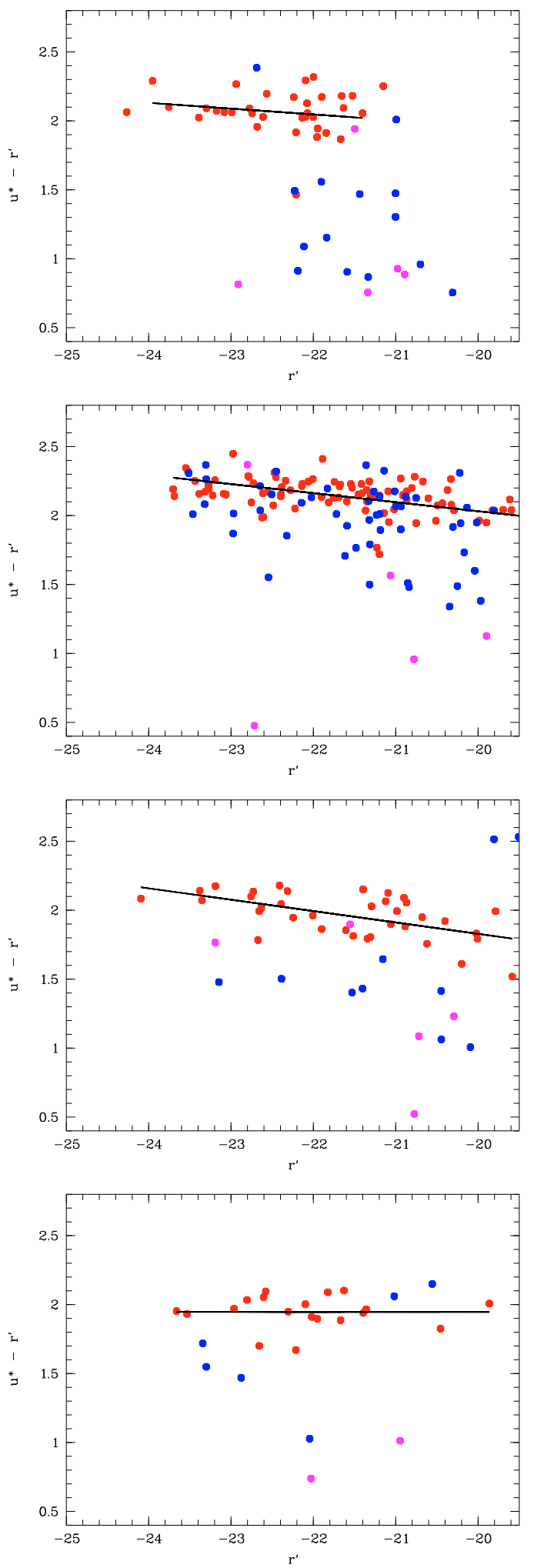

Fig. 16. Rest frame $u^{*}-r^{\prime}$ versus absolute $r^{\prime}$ magnitude relation for clusters using spectroscopic redshifts to compute absolute magnitudes. We only plot cluster members in these figures. From top to bottom, figures are for the most luminous, the luminous, the moderately luminous, and the $\mathrm{C} 0$ clusters. Red symbols correspond to early-type galaxies $(T \leq 21)$, blue symbols correspond to late-type non-starburst galaxies ( $58 \geq T \geq 21$ ), and pink symbols correspond to starburst galaxies $(T \geq 59)$. Black continuous lines are computed using only $T \leq 21$ galaxies (see Table 6 ) 
Table 6. Slopes of the red sequences for the four classes of clusters: most luminous, luminous, moderately luminous, and $\mathrm{C}$.

\begin{tabular}{cc}
\hline \hline Category & Slope \\
\hline most luminous & $-0.04 \pm 0.04$ \\
luminous & $-0.04 \pm 0.02$ \\
moderately luminous & $-0.10 \pm 0.04$ \\
C0 & $-0.01 \pm 0.05$ \\
\hline
\end{tabular}

There are potential differences between $\mathrm{C} 0$ clusters (without detectable X-ray emission) and other classes. The slope of the $\mathrm{RS}$ appears nearly flat for $\mathrm{C} 0$ clusters, while it is more negative for more luminous clusters. This effect is only of little significance when considering the uncertainty of the slope of these RS's. We performed a bi-dimensional Kolmogorov-Smirnov statistical test on the early-type galaxies of Fig. 16 though. The probability that the $\mathrm{C} 0$ and the most luminous cluster earlytype galaxies come from the same population is only $0.6 \%$. The probability that the $\mathrm{C} 0$ and the luminous cluster early-type galaxies come from the same population is only $0.1 \%$. Finally, the probability that the $\mathrm{C} 0$ and the moderately luminous cluster early-type galaxies come from the same population is $3.1 \%$. At least for the most luminous and luminous cluster populations, early-type galaxies therefore seem to be differently distributed in a color-magnitude relation compared to $\mathrm{C} 0$ cluster early-type galaxies. If these differences come from the slope of the RS, this effect could be interpreted as a metallicity effect (Kodama $\&$ Arimoto 1997). The more massive a galaxy, the more easily it will retain metals against dissipative processes. The more metals present in a galaxy, the redder the galaxy will be. Massive galaxies are therefore expected to be redder than lower mass objects. A possible explanation would be that the faint early-type $\mathrm{C} 0$ cluster galaxies would originate from depleted cores of larger galaxies, which would make them metal rich before they become faint (see e.g. Adami et al. 2006). This is possible for example in small groups, where the velocity dispersion is low enough to favor galaxy-galaxy encounters.

Galaxy members of the most luminous clusters also appear to exhibit a more pronounced dichotomy between early- and late-type objects. Blue members of the most luminous clusters are clearly bluer than blue members of the less luminous clusters.

\subsubsection{Age of formation of the cluster galaxy stellar populations}

We expect distant clusters to naturally exhibit younger galaxy star populations compared to nearby structures. In order to investigate this question, we computed with LePhare the ages of the stellar population in galaxies with a spectroscopic redshift lying inside the considered clusters. The templates used to generate public photometric redshifts in the CFHTLS does not allow us to provide this information, so we used in LePhare the Bruzual \& Charlot (2003) templates, fixing the redshifts to the spectroscopic values. The metallicity was let free to vary between 0.004 , 0.008 , and $0.02 Z_{\odot}$. In C0 clusters, $z=[0.3 ; 0.6]$ galaxies have a stellar population aged $6.2 \pm 1.9 \mathrm{Gyr}$, and $z=[0.7 ; 0.9]$ galaxies have a stellar population aged only $2.7 \pm 1.3 \mathrm{Gyr}$. Considering members of luminous clusters, $z=[0.25 ; 0.35]$ galaxies have a stellar population aged $7.4 \pm 1.0 \mathrm{Gyr}$, and $z=[0.35 ; 0.65]$ galaxies have a stellar population aged only $5.3 \pm 2.1$ Gyr. Finally, members of the most luminous clusters, $z=[0.4 ; 0.65]$ galaxies have a stellar population aged 5.2 $\pm 2.1 \mathrm{Gyr}$, and $z=[0.75 ; 1.25]$ galaxies have a stellar population aged $3.3 \pm 1.1 \mathrm{Gyr}$.

Taking the mean redshift of the highest redshift bin for each of these three categories and diminishing the corresponding elapsed time since the beginning of the Universe by the mean age of the early-type galaxy stellar populations leads us to estimate the mean age of formation of the star populations in these galaxies. Galaxy stellar populations probably formed at $z \sim 1.6$ in C0 clusters, at $z \sim 2$ in luminous clusters, and at $z \sim 2.5$ in the most most luminous clusters. These values agree well with general expectations for the massive clusters to form earlier than low-mass structures, up to redshifts close to $z \sim 2$.

\subsubsection{Red sequence using photometric redshifts and color-color diagrams}

In order to study larger samples and detect possible weak effects, we used photometric redshifts to define a cluster membership, and compute absolute magnitudes and colors as provided by the CFHTLS data. Given its photometric redshift, a galaxy was assigned to a cluster when it was closer than $500 \mathrm{kpc}$ from the cluster center and at less than 0.08 from the cluster redshift. This corresponds to the values quoted in Table A.1 for cluster galaxies. We were then able to search for RSs in the most luminous, the luminous, and the moderately luminous clusters. Selecting all available clusters in these three categories Fig. 17 clearly shows red sequences in each case. They are all consistent with a $u^{*}-r^{\prime}$ color of 1.9 , the most massive clusters exhibiting the more negative $\mathrm{RS}$ slope (computed with $T \leq 21$ galaxies). On the contrary, the $\mathrm{C} 0$ clusters (no X-ray detection) only exhibit a very low number of early-type galaxies (but still consistent with $\left.u^{*}-r^{\prime} \sim 1.9\right)$. These compact structures therefore appear as quite young structures, with modest early-type galaxy populations.

However, we are merging in Fig. 17 clusters with quite different redshifts, and evolutionary effects could play an important role. We therefore selected only the luminous clusters (the only category providing enough clusters) and divided this population into three different redshifts bins $(\leq 0.3] 0.3,0.5$,$] , and ] 0.5,0.8])$ in Fig. 18. This figure only shows $T \leq 21$ galaxies (early types). The RSs appear very similar, with the most negative slope occuring for $z=] 0.5,0.8$ ] clusters. If evolutionary effects are present, they are therefore rather weak, besides the most distant clusters appearing to have the most negative RS slope $(-0.069 \pm 0.017)$. This is consistent with the slope computed for the most luminous clusters (which are also nearly all at redshift higher than 0.5): $-0.052 \pm 0.015$.

It could be argued that the use of photometric redshifts could introduce a bias owing for example to SEDs not adapted to highdensity regions. In order to check the previous results, we therefore simply draw $u^{*}-r^{\prime}$ versus $r^{\prime}-z^{\prime}$ color-color diagrams for the same sets of clusters. Figure 19 shows that in both cases early types still occupy well defined loci in the color-color space, confirming the existence of an old galaxy population in these cluster classes.

We therefore confirm that both massive and less massive $\mathrm{X}$-ray structures in our sample exhibit quite similar red sequences, making them overall quite old compact structures. Non-X-ray clusters are probably minor structures with a poor spectral early-type population.

Figure 17 also shows a slightly larger percentage of starburst galaxies (as determined during the photometric redshift computation process: see Coupon et al. 2009) in low-luminosity clusters. C0, moderately luminous, and luminous clusters exhibit $20 \%$ more starburst galaxies compared to the most luminous 

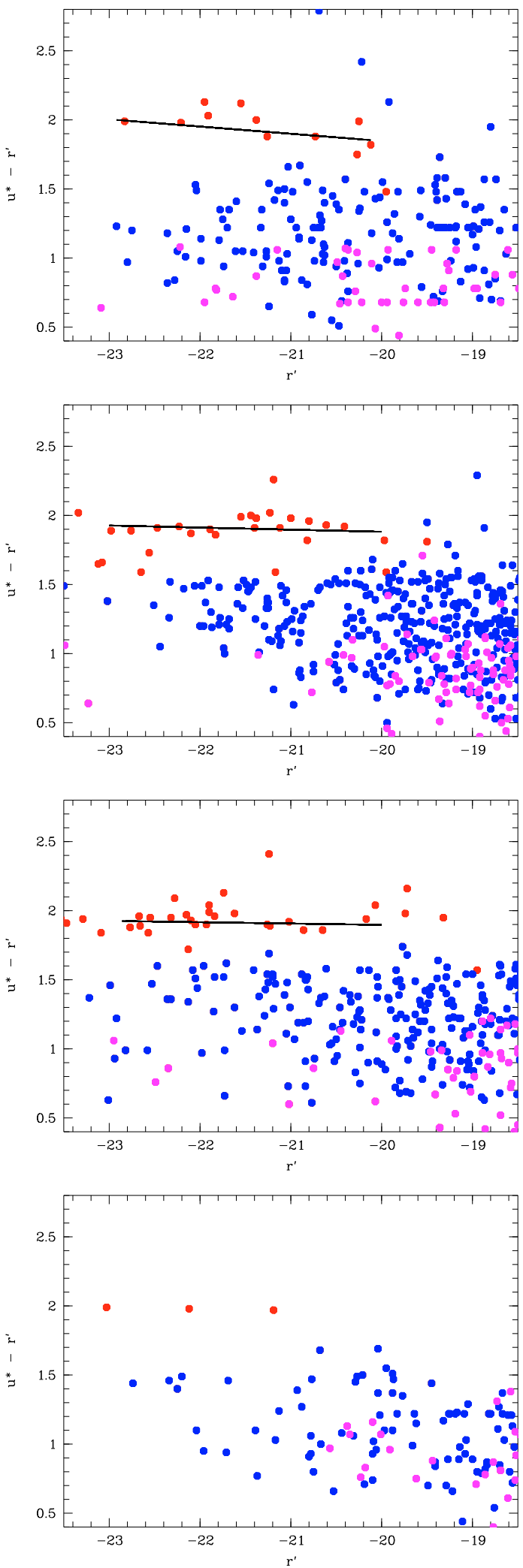

Fig. 17. $u^{*}-r^{\prime}$ versus $r^{\prime}$ with red dots being $T \leq 21$ galaxies (early types), blue dots being $58 \geq T \geq 21$ galaxies (late types), and pink dots being starburst galaxies. From top to bottom, figures are for the most massive, the massive, the moderately massive, and the $z \leq 1 \mathrm{C} 0$ clusters. Absolute magnitude computations are based on photometric redshifts. Black continuous lines are the RSs computed with $T \leq 21$ galaxies, except for $\mathrm{C} 0$ clusters where we had not enough available early-type galaxies.

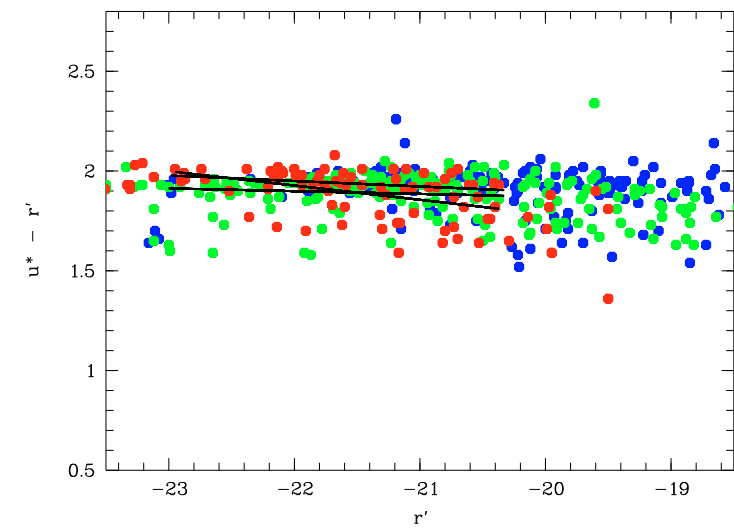

Fig. 18. $u^{*}-r^{\prime}$ versus $r^{\prime}$ for $T \leq 21$ galaxies (early types). Red dots: $z=] 0.5,0.8]$, green dots: $z=] 0.3,0.5]$, and blue dots: $z=[0 ., 0.3]$. Black continuous lines are the RSs computed with $T \leq 21$ galaxies.

clusters. This is an expected behavior, and it agrees qualitatively well for example with Urquhart et al. (2010).

\subsection{Luminosity functions}

In the same spirit, we checked whether our compact structures behave as genuine clusters or groups concerning their galaxy luminosity functions. For a detailed study of the individual XMM-LSS C1 cluster luminosity functions, we refer the reader to Alshino et al. (2010). We computed luminosity functions using galaxies within the cluster bins (according to photometric redshifts). The Schechter function fitting was performed allowing a constant background to take into account galaxies included in the photometric redshift slice, but not part of the clusters.

Selecting all clusters $(\mathrm{C} 1+\mathrm{C} 2+\mathrm{C} 3+\mathrm{C} 0)$, stacking their luminosity functions, and only limiting absolute magnitude to $i^{\prime} \leq-17.5$ in order to not be too affected by incompleteness, we got a best-fit of a Schechter function with alpha $=-1.15 \pm 0.09$ and $M^{*} i^{\prime}=-23.8 \pm 0.8$. This is consistent within error bars with the estimates of Alshino et al. (2010) at $z \sim 0.3$. Applying the same procedure to the $\mathrm{C} 3$ clusters, we get a slightly shallower Schechter fit: alpha $=-0.96 \pm 0.14$ and $M^{*} i^{\prime}=-22.1 \pm 0.6$.

If we use the luminosity categories, we can similarly compute Schechter fits for the luminous and the moderately luminous clusters (most luminous clusters are too distant and therefore undersampled toward the faint magnitudes, and $\mathrm{C} 0$ and $\mathrm{C} 2$ clusters are not numerous enough). We get alpha $=-1.1 \pm 0.03$ and $M^{*} i^{\prime}=-23.4 \pm 0.3$ for the moderately luminous and we get alpha $=-1.1 \pm 0.03$ and $M^{*} i^{\prime}=-23.2 \pm 0.2$ for the luminous clusters.

The fitted Schechter functions agree with those of bona fide clusters at similar depth (e.g. Lumsden et al. 1997). Slopes are also similar within error bars between all cluster classes. C1 clusters seem to exhibit brighter $M_{*}$ than $\mathrm{C} 3$ clusters however, which agrees well with the assumption that $\mathrm{C} 1$ clusters would be older and more massive systems than $\mathrm{C} 3$ clusters.

\section{Peculiar structures in the XMM-LSS}

\subsection{Distant cluster candidates}

Several compact structures with redshifts $\sim 1$ or higher have already been found in Class 1 (Pacaud et al. 2007) or 2 (Bremer et al. 2006). Some other candidates appear among the C2's 

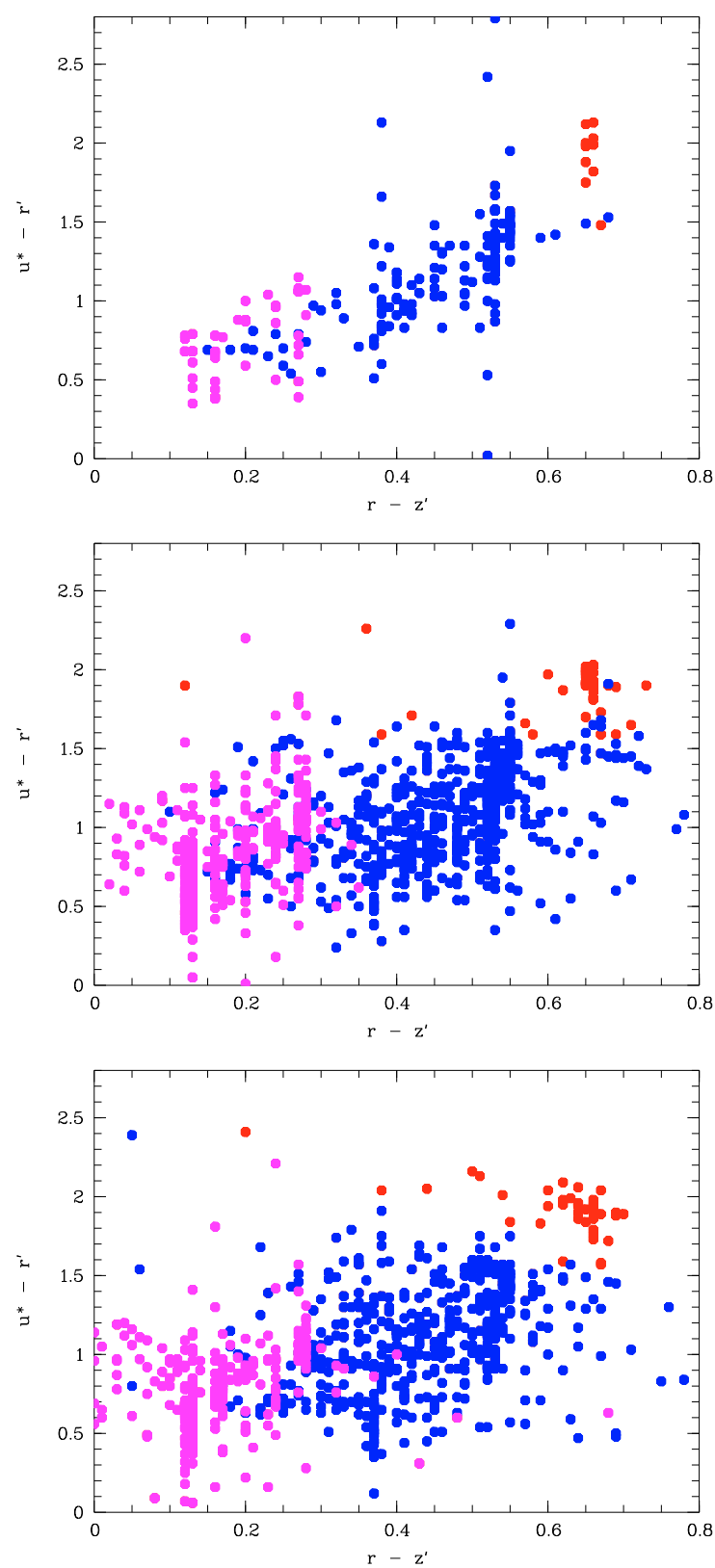

Fig. 19. $u^{*}-r^{\prime}$ versus $r^{\prime}-z$ diagrams with red dots being $T \leq 21$ galaxies (early types), blue dots being $58 \geq T \geq 21$ galaxies (late types), and pink dots being starburst galaxies. The upper figure is for the most luminous, the middle figure is for the luminous, and the lower figure is for the moderately luminous clusters.

(e.g. XLSS J022756.3-043119 at $z \sim 1$ ) and the C0's (022550.4044500 at $z \sim 1.53$ ). This last structure (Fig. 20) is just below the X-ray detection limit. It has an extension of $\sim 13$ arcsec and its extension maximum likelihood is $\sim 10$. We note that the measured flux is $0.2 \pm 0.1 \times 10^{-14} \mathrm{erg} / \mathrm{s} / \mathrm{cm}^{2}$. At $z=1.53$ and for a temperature of $1.5 \mathrm{keV}$, this would lead to an X-ray luminosity of $8.6 \mathrm{e}+43 \mathrm{erg} / \mathrm{s}$.

The weakness of the evidences for an X-ray detection leads us to classify this source as $\mathrm{C} 0$ however, and then to investigate it from the optical side. The regular CFHTLS photometric redshifts (based on $u^{*} g^{\prime} r^{\prime} i^{\prime} z^{\prime}$ magnitudes) are not well suited to study this potential structure because of a lack of near-infrared photometric bands. This candidate is however included in the WIRDS survey (near infrared imaging from CFHT-WIRCAM).

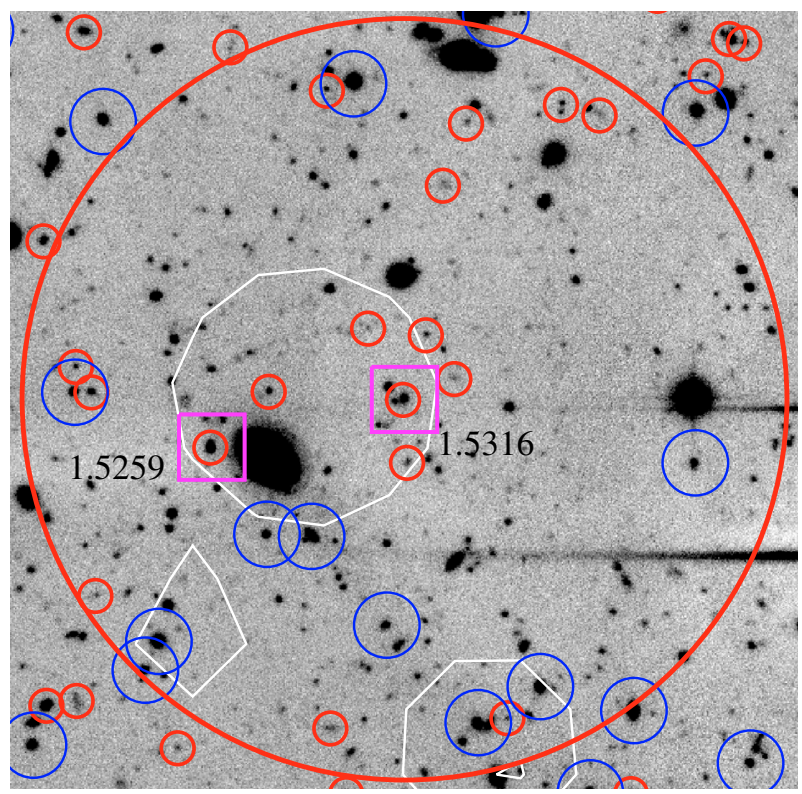

Fig. 20. Distant cluster candidate at $z=1.53$. The large red circle is a $500 \mathrm{kpc}$ radius circle. Blue circles are galaxies with spectroscopic redshifts outside the $z=[1.52 ; 1.54]$ interval. The two magenta squares are the two known spectroscopic redshifts inside the $z=[1.52 ; 1.54]$ interval. Small red circles are the near-infrared-based photometric redshifts inside the $z=[1.43 ; 1.63]$ interval. White contours are the XMM-LSS contours.

Photometric redshifts were computed combining these near infrared data (McCracken et al., private communication) and the CFHTLS deep magnitudes. Figure 20 shows a clear concentration of $z=[1.43 ; 1.63]$ galaxies inside the XMMLSS contours. We therefore may have detected one of the most distant known clusters of galaxies. A near infrared spectroscopic follow up of this candidate is mandatory however to confirm the nature of this very weak X-ray source.

\subsection{Compact structures with discrepant optically and $X$-ray contents}

XLSSC 000 is a C0 structure not detected in the X-rays. Its velocity dispersion is relatively large $\left(435 \pm 88 \mathrm{~km} \mathrm{~s}^{-1}\right)$ however. The Serna-Gerbal analysis does not detect any sign of substructures with the 11 known spectroscopic redshifts, so this velocity dispersion does not appear as obviously biaised. The photometric redshift distribution also presents excesses at the structure redshift. Finally this structure is populated with a significant number of early-type galaxies: among the 21 objects within the $z=0.49$ photometric redshift slice, 9 have type $T \leq 10$. The optical content is therefore similar to what we could expect if we were considering a massive cluster. This case with clear discrepancies between X-ray and optical content remains quite puzzling, and both deeper X-ray observations and additional spectroscopic followup are required to explain the observed behavior.

We have also detected a prominent X-ray structure that is much less evident in optical and that could be a fossil group (XLSSU J021754.6-052655). Described for example in Jones et al. (2003) or Mendes de Oliveira et al. (2006, and references therein), these structures are considered to be the ultimate stage of group evolution: the nearly complete fusion of all bright and intermediate magnitude galaxies of the group into a single bright galaxy. The resulting galaxy is brighter than the 


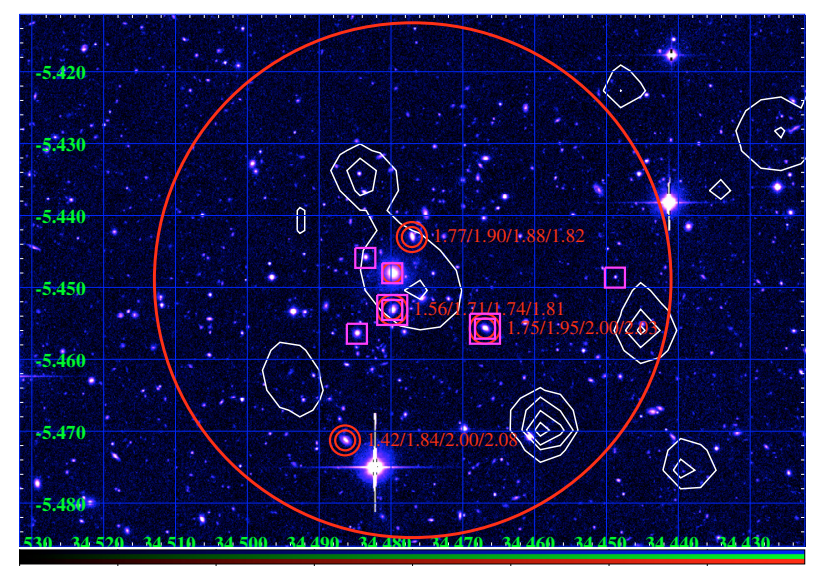

Fig. 21. CFHTLS $i^{\prime}$ band image of the XLSSU J021754.6-052655 XMM-LSS source. White contours are XMM X-ray emission. Pink squares are galaxies with a spectroscopic redshift inside the structure. Red circles are galaxies with a photometric redshift at less than 0.15 from the structure redshift. Large symbols (circles or squares) are galaxies not satisfying the 2 magnitudes criteria in $g^{\prime}, r^{\prime}, i^{\prime}$, or $z^{\prime}$ bands. For these objects we also give the magnitude difference with the brightest galaxy in $g^{\prime} / r^{\prime} / i^{\prime} / z^{\prime}$.

second remaining group galaxy (within half the projected virial radius) by at least 2 mag (in the $R$ band). However, the extended X-ray gas envelope is still present and more luminous than $10^{42} h_{50}^{-2} \mathrm{erg} \mathrm{s}^{-1}$ (Jones et al. 2003). The origin of these structures is still widely debated though. They could find their origin in the small impact parameter of $L \sim L_{*}$ galaxies traveling along filaments (e.g. D’Onghia et al. 2005), or simply in their highly isolated status (e.g. Adami et al. 2007a) so that no galaxies will then have fallen into them lately.

In our survey, XLSSU J021754.6-052655 (classified as C2) is quite similar to these fossil groups. Figure 21 shows the field covered by this galaxy structure. The X-ray source is clearly extended. Available spectroscopic redshifts only show two galaxies at the structure redshift, which are only slightly too bright to satisfy the 2 mag criteria (one is satisfying the criterion in $i^{\prime}$ and $z^{\prime}$ band). Photometric redshifts from Coupon et al. (2009) also exhibit only two other similar galaxies at less than 0.15 from the structure redshift. Considering error bars on magnitude, the magnitude difference between the brightest galaxy and the second brightest object could be consistent with the requested 2 mag gap at the $3-\sigma$ level. We therefore conclude that this object is similar to the structure described in Ulmer et al. (2005) and is very close to the fossil group status.

We investigate if this group is the dominant structure of its cosmological bubble (similarly to Adami et al. 2007a). For this, we selected all known spectroscopic redshifts in the range $[0.241,0.261]$ and at less than $1.5 \mathrm{deg}$ from the group (about $20 \mathrm{Mpc}$ at the structure redshift, close to the average size of known voids: e.g. Hoyle \& Vogeley 2004). Contrary to the results of Adami et al. (2007a), our group does not appear as an isolated structure (Fig. 22). The ratio of galaxies with a spectroscopic redshift inside and outside the range [0.241,0.261] is not significantly different when considering the $1.5 \mathrm{deg}$ region or the complete spectroscopic sample.

\section{Conclusions}

Starting from known XMM-LSS sources, we considered 75 of them for which at least two spectroscopic redshifts were

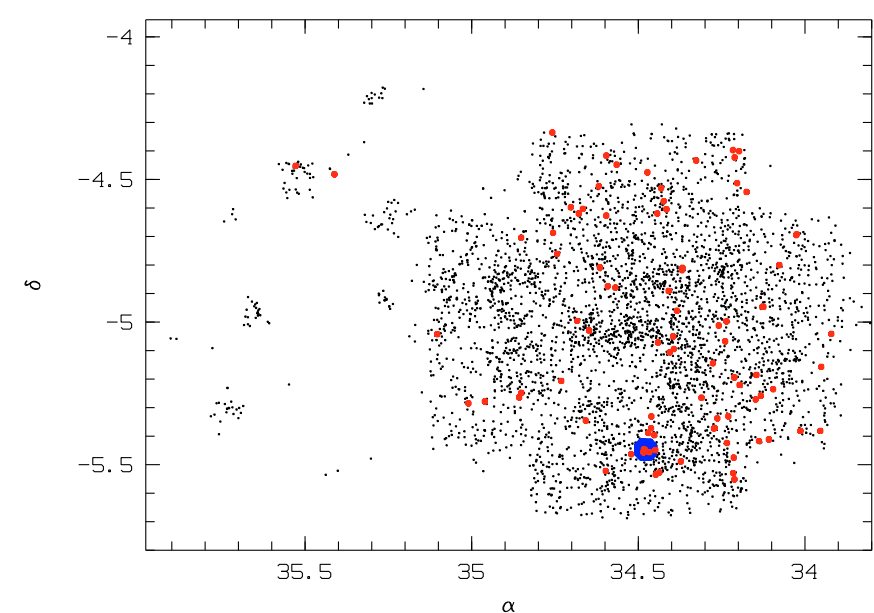

Fig. 22. $\alpha, \delta$ map of the immediate vicinity of XLSS J021754.6-052655. Black dots are all galaxies with a known spectroscopic redshift in a $1.5 \mathrm{deg}$ radius region (large blue filled circle). Red dots are galaxies in the redshift range [0.241,0.261].

available within the $\mathrm{X}$-ray isophotes. We then generated a cata$\log$ of 59 groups or clusters of galaxies in the $z=[0.05 ; 1.53]$ redshift range associated with an X-ray source as well as seven other real compact structures for which X-ray association is not clear. Finally, 11 redshift structures (named C999) detected along the various lines of sight were detected in addition of the main systems and are listed in Appendix. In three cases the X-ray sources are indeed associated with QSOs identified from their optical spectra.

The assessment of the clusters and groups as actual massive structures was based on various spectroscopic data (including PI observations) associated to photometric data from the CFHTLS T0004 release (when available) and some PI data. The analysis (without a priori knowledge of their X-ray class) of the optical lines of sight centered on the X-ray emission was based on criteria such as compactness in redshift space (spectroscopic and photometric), and significant excess in galaxy density obtained within photometric redshift slices and final visual inspection.

All detected systems exhibit "bona fide" clusters or groups optical properties in terms of red sequence, color-color clumping, luminosity function, and morphological segregation. Considering X-ray luminosity classes does not change the results. From the X-ray and optical properties of the compact structures now associated with the XMM extended sources, the $\mathrm{C} 1$ clusters can be considered in most cases as relatively nearby, $\mathrm{X}$-ray bright and optically rich and regular (no sub-clustering) clusters, while $\mathrm{C} 3 \mathrm{~s}$ appear faint and poor at the same redshift and quite rich at high redshift. $\mathrm{C} 2 \mathrm{~s}$ are a mix with the exception of some distant possible candidates. Finally, looking at larger scales using the CFHTLS-W, these clusters statistically appear as clear nodes of the galactic cosmic web, reinforcing therefore their true existence. The full sample of X-ray clusters with associated optical spectroscopic data is available via the L3SDB database (http://13sdb.in2p3.fr:8080/13sdb/). The optical images as well as the details of the redshift determination for all clusters presented in this article will also be publicly available at this place.

Finally, we investigated the photometric redshift precision in our sample as a function of the environment and of the galaxy spectral types (see appendix). We show for example that the 
galaxy photometric redshift accuracy is degraded in the most massive clusters for early- and late-type galaxies.

Acknowledgements. The authors thank R. Bielby, J. Coupon, Y. Mellier, and H.J. McCracken for help. A.D., T.S. and J.S. acknowledge support from the ESA PRODEX Programme "XMM-LSS", from the Belgian Federal Science Policy Office and from the Communauté francaise de Belgique - Actions de recherche concertées - Académie universitaire Wallonie-Europe. H.Q. acknowledges the support of FONDAP Center for Astrophysics 15010003. G.G. is supported by FONDECYT 1085267. The authors thank the referee for useful and constructive comments.

\section{Appendix A: Photometric redshifts in dense environments}

A by-product of the present paper is the test of photometric redshift precision in dense environments. Photometric redshift technique is widely used for several cosmological purposes and is mainly based on synthetic energy distributions (SEDs hereafter) fits to observed magnitudes. The available SEDs in the literature are however mainly selected in low-density environments, outside clusters. Applying these SEDs to cluster galaxies is then potentially problematic. Several papers (e.g. Adami et al. 2008) seem to show various photometric uncertainties as a function of the galaxy spectral-type in these dense environements. If confirmed, this could be due in massive structures to environmental effects driving peculiar color galaxy evolutions. Degeneracies could then be induced between photometric redshift value and galaxy spectral-type when applying classical photometric redshift codes as LePhare or HyperZ (Bolzonella et al. 2000). However, these tendencies are still based on very sparse samples for clusters of galaxies and before embarking on the very demanding task of building cluster-dedicated SEDs, we have to put on a firmer ground the photometric redshift uncertainty variation as a function of the environment and of the galaxy spectral-type.

The XMM-LSS survey offers such a unique opportunity, providing both X-ray and optical characterizations of the clusters, and photometric redshift informations from the CFHTLS. We selected all spectroscopic redshifts included in the present clusters and located in the $500 \mathrm{kpc}$ (radius) central area. This ensures that we have galaxies really located in the densest areas of the clusters. Then we extracted informations (photometric redshift itself and spectral-type) from the CFHTLS T0004 photometric redshift release. Finally, we separately considered clusters brighter than $10^{44} \mathrm{erg} / \mathrm{s}$, between $10^{43}$ and $10^{44} \mathrm{erg} / \mathrm{s}$, and fainter than $10^{43} \mathrm{erg} / \mathrm{s}$. We also considered the $\mathrm{C} 0$ class, acting as the low mass cluster category (we recall that these compact structures are real but without clear X-ray emission).

\section{A.1. General agreement between spectroscopic and photometric redshifts}

We first checked that the general agreement between spectroscopic and photometric redshifts was acceptable inside clusters of galaxies. Figure A.1 shows a good agreement. Selecting a priori galaxies with a $\left|z_{\text {phot }}-z_{\text {spec }}\right| \leq\left(0.15 \times\left(1+z_{\text {spec }}\right)\right)$, the whole cluster galaxy sample exhibits a $\sigma$ of 0.06 . This is only slightly higher than the estimates of Coupon et al. (2009) for the whole CFHTLS W1 field. This shows that from a general point of view, CFHTLS T0004 photometric redshifts are not necessarily worse in clusters than in the field. We have now to investigate in more detail the behavior of the photometric redshift uncertainty as a function of the galaxy spectral-type and as a function of the cluster characteristics.

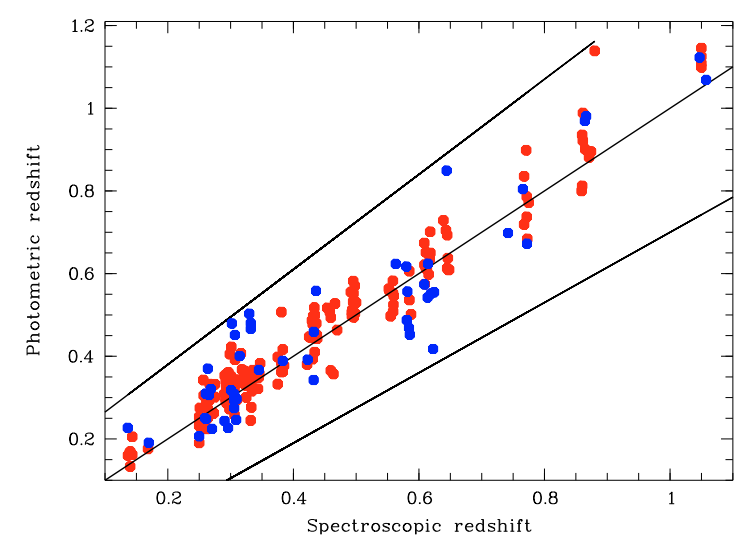

Fig. A.1. Photometric versus spectroscopic redshifts for the cluster galaxies in our sample. Black lines give the perfect relation of slope 1 and the $\pm 0.15 \times(1+z)$ classical uncertainty (see e.g. Ilbert et al. 2006). Red filled circles are early-spectral-type galaxies and blue filled circles are late spectral-type galaxies (see text).

Table A.1. $\sigma$ between photometric and spectroscopic redshifts as a function of the environment and of the galaxy-spectral-type.

\begin{tabular}{cccc}
\hline \hline Cluster class & Early & Late & $\%$ of early-types \\
\hline most luminous & 0.081 & 0.092 & $69 \%$ \\
luminous & 0.036 & 0.082 & $81 \%$ \\
moderately luminous & 0.047 & 0.069 & $72 \%$ \\
C0 & 0.043 & 0.064 & $75 \%$ \\
Global cluster sample & 0.048 & 0.096 & $76 \%$ \\
\hline
\end{tabular}

Notes. The last column indicates the percentage of spectral types $T \leq$ 21 galaxies.

\section{A.2. Photometric redshifts in dense environments and galaxy-spectral-types}

We repeated the previous analysis splitting our samples into early and late-type galaxies and considering the $\mathrm{C} 0$, the most luminous, the luminous, and the moderately luminous clusters. Table A. 1 gives the values of $\sigma$ (computed in the same way as in the previous subsection) in these different cases.

We first detect a clear tendency to have higher uncertainties in photometric redshift calculations in the most luminous clusters. Second, late-type galaxies in luminous and moderately luminous clusters (as well as in $\mathrm{C} 0$ clusters) also exhibit higher uncertainties than early-type galaxies, by a factor of 2 in luminous clusters and by $50 \%$ in moderately luminous and C0 clusters. This behavior was already detected in Guennou et al. (2010).

This can be explained if galaxies were undergoing peculiar evolutions in clusters of galaxies, depending on the mass of the considered clusters, making them different from field galaxies. This would occur for all galaxy types in the most massive clusters, while less massive clusters would only affect late-type galaxies. These various environments do not seem to strongly affect the percentage of early-type galaxies, which stays high anyway (see Table A.1). A finer analysis shows however, as expected, a regular increase of the mean type of $T \leq 21$ galaxies, from 1.4 for the most luminous clusters, to 2.2 for the luminous clusters, and finally to 3.0 for the moderately luminous clusters.

As a conclusion, we can then say that photometric redshift values are generally correct in clusters of galaxies of the present sample (as compared to field environments). However, all galaxies in the most massive clusters and late-type galaxies in all other 
Table B.1. Main other real groups (C999) detected along the lines of sight.

\begin{tabular}{cccccccccc}
\hline \hline XLSSC PH & $\begin{array}{c}\text { RA } \\
\text { deg }\end{array}$ & $\begin{array}{c}\text { Dec } \\
\text { deg }\end{array}$ & $N$ & ZBWT ERRZ & $\begin{array}{c}\text { SIG } \\
\mathrm{km} \mathrm{s}^{-1}\end{array}$ & $\begin{array}{c}\text { ERR } \\
\mathrm{km} \mathrm{s}^{-1}\end{array}$ \\
\hline 065 & 1 & 34.245 & -4.821 & 3 & 0.138 & & & \\
039 & 0 & 35.098 & -2.841 & 3 & 0.183 & & & \\
044 & 1 & 36.141 & -4.234 & 11 & 0.317 & 0.001 & 410 & 87 \\
- & 1 & 36.424 & -4.410 & 4 & 0.142 & & & \\
- & 1 & 36.424 & -4.410 & 4 & 0.632 & & & \\
- & 1 & 36.424 & -4.410 & 3 & 0.915 & & & \\
- & 1 & 36.460 & -4.750 & 17 & 0.885 & & & \\
- & 1 & 36.698 & -4.241 & 3 & 0.210 & & & \\
- & 1 & 36.698 & -4.241 & 3 & 0.432 & & & \\
- & 1 & 36.698 & -4.241 & 3 & 0.705 & & & \\
013 & 1 & 36.858 & -4.538 & 9 & 0.254 & 0.001 & 346 & 75 \\
\hline
\end{tabular}

clusters have their photometric redshift uncertainty increased by a factor of 50 to $100 \%$. Depending on the science goals, this can significantly affect the cluster population definition by photometric redshift criteria, for example for galaxy luminosity function purposes. It would therefore be useful to create clusterdedicated spectroscopic SEDs.

\section{Appendix B: Additional redshift structures}

As a bonus of the general cluster detection process, for a given line of sight other real galaxy groups are detected besides the ones associated with the X-ray emission (C999: see Table B.1). So, if the identification with an optical group would appear wrong in the future, or if more data become available, trace is kept to re-examine other possibilities.

\section{References}

Adami, C., Mazure, A., Ilbert, O., et al. 2005, A\&A, 443, 805 Adami, C., Scheidegger, R., Ulmer, M. P., et al. 2006, A\&A, 459, 679 Adami, C., Russeil, D., \& Durret, F. 2007a, A\&A, 467, 459 Adami, C., Durret, F., \& Mazure 2007b, A\&A, 462, 411 Adami, C., Ilbert, O., Pelló, R., et al. 2008, A\&A, 491, 681 Adami, C., LeBrun, V., Biviano, A., et al. 2009, A\&A, 507, 1225 Adami, C., Durret, F., Benoist, C., et al. 2010, A\&A, 509, A81 Alshino, A., Khosroshahi, H., Ponman, T., et al. 2010, MNRAS, 401, 941 Beers, T., Flyn, K., \& Gebhardt, K. 1990, AJ, 119, 2645 Biviano, A., Katgert, P., Mazure, A., et al. 1997, A\&A, 321, 84 Bohringer, H., et al. 1998, in Wide Field Surveys in Cosmology, ed. S. Colombi, Y. Mellier, \& B. Raban (Paris: Editions Frontières), Proc. 14th IAP Conf., 261
Bolzonella, M., Miralles, J.-M., \& Pelló, R. 2000, A\&A, 363, 476 Boué, G., Durret, F., Adami, C., et al. 2008, A\&A, 489, 11 Bremer, M. N., Valtchanov, I., Willis, J., et al. 2006, MNRAS, 371, 1427 Bruzual, G., \& Charlot, S. 2003, MNRAS, 344, 1000

Carlberg, R. G., Yee, H. K. C., Ellingson, E., et al. 1996, ApJ, 462, 32 Castander, F. J., Bower, R. G., Ellis, R. S., et al. 1995, Nature, 377, 39 Collins, C. A., Burke, D. J., Romer, A. K., Sharples, R. M., \& Nichol, R. C. 1997, ApJ, 479, L117

Coupon, J., Ilbert, O., Kilbinger, M., et al. 2009, A\&A, 500, 981 De Grandi, S., Bohringer, H., Guzzo, L., et al. 1999, ApJ, 514, 148 D’Onghia, E., Sommer-Larsen, J., Romeo, A. D., et al. 2005, ApJ, 630, L109

Dunkley, J., Komatsu, E., Nolta, M. R., et al. 2009, ApJS, 180, 306 Ebeling, H., Edge, A. C., Bohringer, H., et al. 1998, MNRAS, 301, 881 Edge, A. C., \& Stewart, G. C. 1991, MNRAS, 252, 428

Finoguenov, A., Guzzo, L., Hasinger, G., et al. 2007, ApJS, 172, 182 Finoguenov, A., Connelly, J. L., Parker, L. C., et al. 2009, ApJ, 704, 564 Finoguenov, A., Watson, M. G., Tanaka, M., et al. 2010, MNRAS, 403, 2063 Franzetti, P., Scodeggio, M., Garilli, B., et al. 2007, A\&A, 465, 711 Gavazzi, R., \& Soucail, G. 2007, A\&A, 462, 459

Gandhi, P., Garcet, O., Disseau, L., et al. 2006, A\&A, 457, 393

Guennou, L., Adami, C., Ulmer, M. P., et al. 2010, A\&A, 523, A21

Hamana, T., Miyazaki, S., Kashikawa, N., et al. 2009, PASJ, 61, 833

Henry, J. P. 1997, ApJ, 489, L1

Hoyle, F., \& Vogeley, M. S. 2004, ApJ, 607, 751

Ilbert, O., Tresse, L., Zucca, E., et al. 2005, A\&A, 439, 863

Ilbert, O., Arnouts, S., McCracken, H. J., et al. 2006, A\&A, 457, 841

Jones, L. R., Scharf, C., Ebeling, H., et al. 1998, ApJ, 495, 100

Jones, L. R., Ponman, T. J., Horton, A., et al. 2003, MNRAS, 343, 627

Kodama, T., \& Arimoto, N. 1997, A\&A, 320, 41

Le Fèvre, O., Vettolani, G., Garilli, B., et al. 2005, A\&A, 439, 845

Lumsden, S. L., Collins, C. A., Nichol, R. C., et al. 1997, MNRAS, 290, 119

Mazure, A., Adami, C., Pierre, M., et al. 2007, A\&A, 467, 49

Mendes de Oliveira, C., Cypriano, E. S., Sodré Jr., L. 2006, AJ, 131, 158

Pacaud, F., Pierre, M., Refregier, A., et al. 2006, MNRAS, 372, 578

Pacaud, F., Pierre, M., Adami, C., et al. 2007, MNRAS, 382, 1289

Pierre, M., Valtchanov, I., Altieri, B., et al. 2004, JCAP, 9, 11

Pierre, M., Pacaud, F., Duc, P.-A., et al. 2006, MNRAS, 372, 591

Pierre, M., Chiappetti, L., Pacaud, F., et al. 2007, MNRAS, 382, 279

Quintana, H., \& Melnick, J. 1982, AJ, 87, 972

Rizzo, D., Adami, C., Bardelli, S., et al. 2004, A\&A, 413, 453

Romer, A. K., Collins, C. A., Bohringer, H., et al. 1994, Nature, 372, 75

Romer, A. K., Nichol, R. C., Holden, B. P., et al. 2000, ApJS, 126, 209

Romer, A. K., Viana, P. T. P., Liddle, A. R., \& Mann, R. G. 2001, ApJ, 547, 594

Rosati, P., Della Ceca, R., Norman, C., \& Giacconi, R. 1998, ApJ, 492, L21

Serna, A., \& Gerbal, D. 1996, A\&A, 309, 65

Smith, B. W., Mushotzky, R. F., \& Serlemitsos, P. J. 1979, ApJ, 227, 37

Stanford, S. A., Holden, B., Rosati, P., et al. 2002, AJ, 123, 619

Ueda, Y., Watson, M., Stewart, G., et al. 2008 ApJS, 179, 124

Ulmer, M. P., Adami, C., Covone, G., et al. 2005, ApJ, 624, 124

Urquhart, S. A., Willis, J. P., Hoekstra, H., \& Pierre, M. 2010, MNRAS, 406, 368

Vikhlinin, A., McNamara, B. R., Forman, W., et al. 1998, ApJ, 502, 558

Werner, N., Finoguenov, A., Kaastra, J. S., et al. 2008, A\&A, 482, L29

Yee, H. K. C., \& Ellington, E. 2003, ApJ, 585, 226 